\title{
Solution of the Bethe-Salpeter equation for pion-nucleon scattering
}

\author{
A. D. Lahiff and I. R. Afnan \\ Department of Physics, The Flinders University of South Australia, \\ GPO Box 2100, Adelaide 5001, Australia
}

(June 25, 2018)

\begin{abstract}
A relativistic description of pion-nucleon scattering based on the fourdimensional Bethe-Salpeter equation is presented. The kernel of the equation consists of $s$ - and $u$-channel nucleon and $\Delta(1232)$ pole diagrams, as well as $\rho$ and $\sigma$ exchange in the $t$-channel. The Bethe-Salpeter equation is solved by means of a Wick rotation, and good fits are obtained to the $s$ - and $p$-wave $\pi N$ phase shifts up to $360 \mathrm{MeV}$ pion laboratory energy. The coupling constants determined by the fits are consistent with the commonly accepted values in the literature.
\end{abstract}

\section{INTRODUCTION}

Pion-nucleon $(\pi N)$ scattering is an important example of a strong interaction, and as such plays a significant role in many nuclear reactions involving pions, the most interesting example in recent years being pion photoproduction. It is generally accepted that the fundamental theory of strong interactions is Quantum Chromodynamics (QCD), and therefore a theory describing $\pi N$ scattering should ideally be derived from QCD. However, due to the non-perturbative nature of confinement, QCD has not been amiable to solutions for low and intermediate energies, and hence it is necessary to use an effective theory. In principle the effective theory should be as close as possible to the fundamental one, and so should satisfy the same symmetries, in particular chiral symmetry, which is known to be important for low-energy physics. Therefore, in place of the QCD Lagrangian a chirally-invariant hadronic Lagrangian is used, where the degrees of freedom are mesons and baryons rather than quarks and gluons. For low energies it is expected that the detailed quark structure of hadrons is relatively unimportant, and that it is only at very high energies that explicit quark degrees of freedom are essential. The great success of meson-exchange models for nucleon-nucleon $(N N)$ scattering in the description of $N N$ phase shifts is an example of this.

A number of dynamical models of $\pi N$ scattering have been developed over the past few years. Most begin with a potential which is iterated in a Lippman-Schwinger-type equation to give the scattering amplitude, from which the phase shifts and observables are obtained. This method ensures that two-body unitarity is respected, and that multiple scattering effects are taken into account. The simplest models use separable potentials [1 [0 [n, in which the parameters have no physical meaning, and furthermore, different sets of parameters are used in each partial wave. While these models provide good descriptions of the $\pi N$ phase shifts, they provide no information about the interaction process. An alternative is to derive a potential from a Lagrangian which describes the couplings between the various 
mesons and baryons. In tree-level models [6 [1]], the potential is unitarized using the $K$ matrix approximation, which relies on the assumption that for low energies the $K$-matrix is equal to the potential. While this method can provide a good description of the $\pi N$ data at low energies, in order to cover a larger region of energies and to be able to investigate the nature of resonances, the potential must be iterated to all orders. This has been done recently [11 18] in models based on three-dimensional (3-D) approximations to the BetheSalpeter equation [19]. Phenomenological form factors are required at the vertices in order to provide convergence, and so in general more free parameters are needed than in tree-level models, which can give good results without any form factors [8].

There are several other approaches to $\pi N$ scattering in the literature which give results of a similar quality. The meson-exchange model of Jäde [20] uses the solitary boson exchange method to regularize self-energy diagrams instead of using form factors, and gives good descriptions of both $\pi N$ and $N N$ scattering with the same set of parameters. Sato and Lee constructed a meson-exchange model using an effective Hamiltonian [21], while Ellis and Tang [22] used chiral perturbation theory. Quark models have also been used to describe $\pi N$ scattering [23 25]. Fuda [4] developed a Poincaré invariant front form model of $\pi N$ scattering, in which the potentials were assumed to be seperable. This model was later extended [5] to a pion laboratory kinetic energy of $1 \mathrm{GeV}$, giving an excellent description of the phase shifts and inelasticities in the $s$-, $p$ - and $d$-waves.

The exact $\pi N \leftarrow \pi N$ amplitude for a given Lagrangian can in principle be obtained from the full BS equation, with fully dressed propagators in the $\pi N$ intermediate state, and a potential consisting of all 1- and 2-particle irreducible connected diagrams. By definition this exact amplitude would satisfy both crossing and chiral symmetry, and have the correct one-body limit (an equation is said to have the one-body limit if it correctly reduces to either the Klein-Gordon or Dirac equation if the mass of either of the particles becomes infinitely heavy). Unfortunately, at present it is impossible to construct the potential for the full BS equation, as it would contain an infinite number of Feynman diagrams. Consequently the potential and propagators must be approximated in some way. The simplest and most commonly used approximation involves replacing the dressed propagators in the intermediate states with bare propagators with poles at the physical masses, and truncating the potential so that it contains only the lowest-order diagrams, i.e. the tree-level diagrams. The resulting equation is generally referred to as the ladder BS equation. There are some problems with this equation, such as it does not have the correct one-body limit [26]. Also some symmetries present in the approximate potential, such as crossing and chiral symmetry, may be violated in the solution of the BS equation [27].

The BS equation for the $\pi N$ scattering amplitude is a covariant four-dimensional integral equation, in which the integration is over the relative energy and relative momentum of the $\pi N$ intermediate state. It is the presence of the relative energy as an integration variable that is responsible for the complicated singularity structure of the kernel. Frequently the BS equation is reduced to a 3-D integral equation in order to avoid the difficulties involved in the handling of the singularities of the kernel. This is achieved by approximating the kernel in such a way that the integration over the relative energy can be carried out explicitly, resulting in a 3-D integral equation. There are an infinite number of 3-D reductions of the BS equation [28], all of which satisfy relativistic elastic two-body unitarity. There is no overwhelming reason to choose one particular 3-D approximation over any other. Some of the 
3-D equations are chosen so as to overcome problems caused by approximating the full BS equation with the ladder approximation. For example, the "smooth propagator" [29], used by Pearce and Jennings [11] and Jäde [20], attempts to restore chiral symmetry. However, other problems are introduced when the dimensionality is reduced from 4-D to 3-D. Pascalutsa and Tjon [17] have shown that when the nucleon self-energy is calculated using most of the commonly used 3-D propagators, there are differences in the renormalization between the positive and negative energy states, which is an indication that charge conjugation and $\mathrm{CPT}$ symmetries are violated. Another problem is that there are significant differences in the half-off-shell amplitudes as calculated by different 3-D reductions of the BS equation [14], even when they all give the same results on-shell. This could have significant implications, in that it is the off-shell behaviour that is important when the $\pi N$ amplitude is used as input into the calculation of other nuclear reactions, such as pion-nucleus scattering, pion production in nucleon-nucleon collisions, or pion photoproduction.

In the present investigation we describe a relativistic model of $\pi N$ scattering in which the BS equation is solved directly in four-dimensions. In this way we can avoid the ambiguities encountered in reducing the BS equation to three-dimensions. This we hope will give us coupling constants that could be compared to those extracted from QCD models, as it has been shown that there are considerable differences in coupling constants obtained using 3-D equations as compared to the BS equation [30]. More important is the fact that our $\pi N$ amplitude can be gauged [31] to give a photoproduction amplitude that satisfies unitarity and gauge invariance.

To our knowledge there are currently no models based on the BS equation that give the $s$ and $p$-wave $\pi N$ phase-shifts in good agreement with the empirical data. The only previous meson-exchange model for $\pi N$ scattering to have used the 4-dimensional BS equation was that of Nieland and Tjon [32]. Since the potential consisted only of the $u$-channel nucleon pole diagram, this model could only give the $P_{33}$ phase-shifts in agreement with experiment. In order to get a good description of all the $s$ - and $p$-wave phase shifts, it is necessary to include in the potential $s$ - and $u$-channel $N$ and $\Delta(1232)$ poles, and in addition $t$-channel $\rho$ and $\sigma$ exchange diagrams. All of the recent $\pi N$ meson-exchange models have included these diagrams, although Schütz et al. replaced the $\rho$ and $\sigma$ exchange diagrams with correlated two-pion exchange [15]. The model of Schütz was later extended [16] to include the coupling to the $\pi \Delta, \eta N$ and $\sigma N$ channels, and included the $N^{*}(1535)$ pole diagrams in the potential. Pascalutsa and Tjon included the Roper resonance [17], and later also included the $S_{11}$ and $D_{13}$ resonances as elementary particle poles in the potential [18]. We do not include the Roper or any other higher baryon resonances, or coupling to channels other than $\pi N$, as these contributions are expected to be small for elastic $\pi N$ scattering and at pion energies below the Roper resonance. Here we are mainly interested in $\pi N$ scattering below the two-pion production threshold (around $360 \mathrm{MeV}$ pion laboratory energy).

The organization of this paper is as follows. In Sec. [I] we state the Bethe-Salpeter equation and give expressions for the dressed vertices and propagators. The Lagrangian and form factors used in our model, and the choice of spin-3/2 propagator for the $\Delta(1232)$, are discussed in Sec. III. The renormalization procedure is outlined in Sec. IV, and we then proceed to describe our method of solving the BS equation in Sec. D. Here we also discuss the analytic structure of the BS equation. In Sec. VI we present results of fits to the SM95 partial wave analysis of Arndt et al. [33] from threshold up to $360 \mathrm{MeV}$ pion laboratory 
energy. We compare the coupling constants obtained in the present work to those obtained using models of $\pi N$ scattering based on three-dimensional reductions of the BS equation. We also discuss how the phase shifts are built up from the individual diagrams in the potential, and calculate the renormalized pion cutoff mass in order to examine the effect of dressing on the $\pi N N$ form factor. Next we consider a second model which differs from the first in the parameterization of the form factors. We conclude this section by presenting results for the phase shifts up to $600 \mathrm{MeV}$. Finally, in Sec. VII we present some concluding remarks.

\section{THE BS EQUATION FOR $\pi N$ SCATTERING}

We consider the scattering process

$$
\pi\left(p_{\pi}^{\prime}\right)+N\left(p_{N}^{\prime}\right) \longleftarrow \pi\left(p_{\pi}\right)+N\left(p_{N}\right)
$$

where $p_{\pi}$ and $p_{N}$ represent the incoming 4-momenta of the pion and nucleon, while $p_{\pi}^{\prime}$ and $p_{N}^{\prime}$ are the outgoing 4-momenta. The total 4 -momentum $P$ is given by

$$
P=p_{\pi}+p_{N}=p_{\pi}^{\prime}+p_{N}^{\prime}
$$

and the relative 4-momenta in the initial and final states are defined as

$$
q=\frac{1}{2}\left(p_{N}-p_{\pi}\right), \quad q^{\prime}=\frac{1}{2}\left(p_{N}^{\prime}-p_{\pi}^{\prime}\right) .
$$

In the centre-of-mass (CM) frame the total 4-momentum is related to the total energy $\sqrt{s}$ by $P=(\sqrt{s}, \mathbf{0})$. In addition, the Mandelstam variables $u$ and $t$ are given in terms of the relative momenta as

$$
u=\left(q+q^{\prime}\right)^{2}, \quad t=\left(q-q^{\prime}\right)^{2} .
$$

Having defined the kinematics, the Bethe-Salpeter equation [19] for the $\pi N \leftarrow \pi N$ amplitude $T\left(q^{\prime}, q ; P\right)$ can be written as

$$
T\left(q^{\prime}, q ; P\right)=V\left(q^{\prime}, q ; P\right)-\frac{i}{(2 \pi)^{4}} \int d^{4} q^{\prime \prime} V\left(q^{\prime}, q^{\prime \prime} ; P\right) G_{\pi N}\left(q^{\prime \prime} ; P\right) T\left(q^{\prime \prime}, q ; P\right)
$$

where $V$ is the potential. The two-body $\pi N$ propagator $G_{\pi N}$ is the product of the pion and nucleon propagators, i.e.

$$
G_{\pi N}(q ; P)=\frac{1}{(P / 2-q)^{2}-m_{\pi}^{2}+i \epsilon} \frac{\not P / 2+\not 1+m_{N}}{(P / 2+q)^{2}-m_{N}^{2}+i \epsilon},
$$

where $m_{\pi}$ and $m_{N}$ are the charge-averaged pion and nucleon masses. In principle both the nucleon and pion propagators in the $\pi N$ intermediate states should be dressed. However, since here we are only requiring that two-body unitarity be maintained, we have replaced the dressed nucleon and pion propagators with bare propagators with poles at the physical nucleon and pion masses.

The potential $V\left(q^{\prime}, q ; P\right)$ is constructed from the sum of $s^{-}, t$ - and $u$-channel pole diagrams (see Sec. III). It is well known that when an $s$-channel pole is included in the potential of the 
ladder BS equation, or one of its 3-D approximations, the solution of the BS equation also contains an $s$-channel pole diagram, in which the propagator and vertices are dressed [34]. The potential can be divided into the sum of non-pole and pole contributions,

$$
V\left(q^{\prime}, q ; P\right)=V_{\mathrm{NP}}\left(q^{\prime}, q ; P\right)+\sum_{B} \Gamma_{\pi N B}^{(0) \dagger}\left(q^{\prime} ; P\right) d_{B}^{(0)}(P) \Gamma_{\pi N B}^{(0)}(q ; P)
$$

where $\Gamma_{\pi N B}^{(0)}$ is the bare $\pi N B$ vertex, and $d_{B}^{(0)}$ is the bare propagator for baryon $B$. At present the only baryons we include in the potential are the nucleon and $\Delta(1232)$, and so we have $B=N, \Delta$. The pole part of Eq. (7) consists of the sum of $s$-channel baryon pole diagrams while $V_{\mathrm{NP}}$, the non-pole part of the potential, contains the $u$ - and $t$-channel exchange diagrams. With the potential having this form, the solution of the BS equation can be written in a similar way as

$$
T\left(q^{\prime}, q ; P\right)=T_{\mathrm{NP}}\left(q^{\prime}, q ; P\right)+\sum_{B} \Gamma_{\pi N B}^{\dagger}\left(q^{\prime} ; P\right) d_{B}(P) \Gamma_{\pi N B}(q ; P),
$$

where $\Gamma_{\pi N B}$ and $d_{B}$ are the dressed $\pi N B$ vertex and dressed baryon propagator respectively. The non-pole part of the $T$-matrix $T_{\mathrm{NP}}$ is the solution of the BS equation with a potential consisting of the sum of the $u$ - and $t$-channel poles, i.e.

$$
T_{\mathrm{NP}}\left(q^{\prime}, q ; P\right)=V_{\mathrm{NP}}\left(q^{\prime}, q ; P\right)-\frac{i}{(2 \pi)^{4}} \int d^{4} q^{\prime \prime} V_{\mathrm{NP}}\left(q^{\prime}, q^{\prime \prime} ; P\right) G_{\pi N}\left(q^{\prime \prime} ; P\right) T_{\mathrm{NP}}\left(q^{\prime \prime}, q ; P\right) \text {. }
$$

Equations (5), (7), (8) and (9) are shown diagrammatically in Fig. 11.

The dressed $\pi N N$ vertex is given in terms of the bare vertex and non-pole part of the $T$-matrix as (see Fig. 2)

$$
\Gamma_{\pi N N}(q ; P)=\Gamma_{\pi N N}^{(0)}(q ; P)-\frac{i}{(2 \pi)^{4}} \int d^{4} q^{\prime \prime} \Gamma_{\pi N N}^{(0)}\left(q^{\prime \prime} ; P\right) G_{\pi N}\left(q^{\prime \prime} ; P\right) T_{\mathrm{NP}}\left(q^{\prime \prime}, q ; P\right) \text {. }
$$

The bare and dressed nucleon propagators are

$$
\begin{aligned}
d_{N}^{(0)}(P) & =\left[\not P-m_{N}^{(0)}+i \epsilon\right]^{-1} \\
d_{N}(P) & =\left[\not P-m_{N}^{(0)}-\Sigma_{N}(P)+i \epsilon\right]^{-1}
\end{aligned}
$$

where $m_{N}^{(0)}$ is the bare nucleon mass. Also, the nucleon self-energy $\Sigma_{N}(P)$ is given by

$$
-i \Sigma_{N}(P)=-\frac{1}{(2 \pi)^{4}} \int d^{4} q \Gamma_{\pi N N}^{(0)}(q ; P) G_{\pi N}(q ; P) \Gamma_{\pi N N}^{\dagger}(q ; P)
$$

and is illustrated in Fig. 3. Making use of Eq. (10), the nucleon self-energy can be written as

$$
\begin{aligned}
& -i \Sigma_{N}(P)=-\frac{1}{(2 \pi)^{4}} \int d^{4} q \Gamma_{\pi N N}^{(0)}(q ; P) G_{\pi N}(q ; P) \Gamma_{\pi N N}^{(0) \dagger}(q ; P) \\
& \quad+\frac{i}{(2 \pi)^{8}} \int d^{4} q^{\prime} d^{4} q \Gamma_{\pi N N}^{(0)}\left(q^{\prime} ; P\right) G_{\pi N}\left(q^{\prime} ; P\right) T_{N P}\left(q^{\prime}, q ; P\right) G_{\pi N}(q ; P) \Gamma_{\pi N N}^{(0) \dagger}(q ; P) .
\end{aligned}
$$


The first term in the right-hand-side of Eq. (14) corresponds to the one-pion loop dressing of the nucleon, while the second term is the contribution arising from the iteration of the non-pole part of the potential in the BS equation (we refer to this term as the non-pole contribution to the self-energy). Since in the $\pi N$ intermediate states we have approximated the dressed nucleon propagator by a bare nucleon propagator with a pole at the physical nucleon mass, we avoid having to solve a non-linear Schwinger-Dyson equation for the nucleon self-energy.

There are similar expressions to Eqs. (10) and (13) for the dressed $\pi N \Delta$ vertex and the $\Delta$ self-energy, however we do not calculate them explicitly, for reasons given in Sec. IV.

\section{THE POTENTIAL}

The potential, or driving term, which is iterated to all orders in the BS equation to obtain an amplitude satisfying two-body unitarity, consists of all the tree-level Feynman diagrams contributing to the process $\pi N \leftarrow \pi N$, derived from the interaction Lagrangian under consideration. In this section we discuss the Lagrangian used, the coupling constants in this Lagrangian, and the choice of form factors which are necessary to obtain convergence. We also look at the possible different forms of spin-3/2 propagators that can be used for the $\Delta$.

\section{A. The Lagrangian}

The tree-level diagrams shown in Fig. 1 are obtained from the following interaction Lagrangian:

$$
\begin{aligned}
\mathcal{L}_{\text {int }}= & \frac{g_{\pi N N}}{2 m_{N}} \bar{\psi}_{N} \gamma_{5} \gamma^{\mu} \boldsymbol{\tau} \cdot \partial_{\mu} \boldsymbol{\pi} \psi_{N}+\frac{f_{\pi N \Delta}}{m_{\pi}} \bar{\psi}_{\Delta}^{\mu}\left(g_{\mu \nu}+x_{\Delta} \gamma_{\mu} \gamma_{\nu}\right) \mathbf{T} \psi_{N} \cdot \partial^{\nu} \boldsymbol{\pi}+\text { h.c. } \\
& +g_{\rho N N} \bar{\psi}_{N} \frac{1}{2} \boldsymbol{\tau} \cdot\left(\gamma^{\mu} \boldsymbol{\rho}_{\mu}+\frac{\kappa_{\rho}}{2 m_{N}} \sigma^{\mu \nu} \partial_{\mu} \boldsymbol{\rho}_{\nu}\right) \psi_{N}+g_{\rho \pi \pi} \boldsymbol{\rho}^{\mu} \cdot\left(\partial_{\mu} \boldsymbol{\pi} \times \boldsymbol{\pi}\right) \\
& +g_{\sigma N N} \bar{\psi}_{N} \psi_{N} \sigma+\frac{g_{\sigma \pi \pi}}{2 m_{\pi}} \sigma \partial_{\mu} \boldsymbol{\pi} \cdot \partial^{\mu} \boldsymbol{\pi}
\end{aligned}
$$

where $\psi_{N}, \psi_{\Delta}^{\mu}, \boldsymbol{\pi}, \boldsymbol{\rho}_{\mu}$, and $\sigma$ are the fields for the nucleon, delta, pion, rho and sigma, respectively. The derivative couplings of the pion field to the other mesons and baryons ensures that chiral symmetry is satisfied at tree-level. In the $\pi N \Delta$ Lagrangian, $\mathbf{T}$ is the transition operator between isospin-3/2 and $1 / 2$ states, and $x_{\Delta}$ is a parameter that can be adjusted, and its value will be considered when we discuss our choice for the $\Delta$ propagator.

Ideally, all of the coupling constants should be fixed using information from other sources, rather than leaving them as free parameters. Out of all of the coupling constants appearing in the Lagrangian given in Eq. (15), the $\pi N N$ coupling is the best known (although even the $\pi N N$ coupling constant is not without controversy [35, 36]). We use the value advocated

by the Nijmegen group [37], i.e. $g_{\pi N N}^{2} / 4 \pi=13.5$. The remaining coupling constants can be determined in a variety of different ways, but there are some discrepancies.

A value for the $\pi N \Delta$ coupling constant can be obtained by calculating the width for the decay $\Delta \rightarrow \pi N$. The coupling constant is chosen such that the width is equal to its 
experimental value. Assuming that the $\Delta$ self-energy is dominated by the one-pion loop diagram, it is found that $f_{\pi N \Delta}^{2} / 4 \pi=0.36$ gives the correct width. This result makes use of the width calculated on the real $s$-axis, however it has been shown that there are differences between widths calculated on the real axis and in the complex plane [38. A somewhat smaller value for $f_{\pi N \Delta}$ is found using the quark-model relation [39]

$$
f_{\pi N \Delta}^{2}=\frac{72}{25}\left(\frac{m_{\pi}}{2 m_{N}}\right)^{2} g_{\pi N N}^{2}
$$

which gives $f_{\pi N \Delta}^{2} / 4 \pi=0.21$ when the Nijmegen value is used for $g_{\pi N N}$. This smaller value has also been shown to be consistent with the width of the $\Delta, 40$, provided that higher-order mesonic corrections to the $\Delta$ self-energy are included along with the one-pion loop term.

We now consider the $\rho$ exchange diagram, which depends only on the product $g_{\rho}^{2} \equiv$ $g_{\rho \pi \pi} g_{\rho N N}$, as well as $\kappa_{\rho}$, the ratio of the tensor to vector $\rho N N$ coupling constants. There are a number of different ways of determining a value for $g_{\rho}$, as originally discussed by Sakurai [41]. If it is assumed that the isospin-odd $\pi N$ scattering length is dominated by the tree-level $\rho$ exchange diagram, then $g_{\rho}^{2} / 4 \pi=3.1$ is obtained. Alternatively, it can be assumed that the $\rho$ meson couples to both pions and nucleons with the same strength (universality), which means that $g_{\rho}=g_{\rho \pi \pi}=g_{\rho N N}$. An estimation of $g_{\rho \pi \pi}$ can be obtained from the decay $\rho \rightarrow 2 \pi$, which gives $g_{\rho \pi \pi}^{2} / 4 \pi=2.84$. A very similar value of $g_{\rho \pi \pi}^{2} / 4 \pi=2.7$ is given by the Kawarabayashi-Suzuki-Riazuddin-Fayyazuddin (KSRF) relation 42, which is obtained from current algebra and PCAC, and states that $m_{\rho}^{2}=2 g_{\rho \pi \pi}^{2} f_{\pi}^{2}$, where $f_{\pi}=93$ $\mathrm{MeV}$ is the pion decay constant. There is some controversy about the value of $\kappa_{\rho}$. The vector meson dominance model (VMD) gives $\kappa_{\rho}=3.7$ 43, while Höhler and Pietarinen found $\kappa_{\rho}=6.6$ from a $\pi \pi-N \bar{N}$ partial wave analysis 44 .

Although the scalar $\sigma$ meson does not seem to exist in nature, $t$-channel $\sigma$ exchange is included in $\pi N$ models as an effective interaction, representing higher-order processes not explicitly included in the potential, such as correlated $2 \pi$-exchange in the scalar-isoscalar channel. There are therefore no reliable determinations of the mass of the $\sigma$ meson or the magnitudes (or signs) of the $\sigma N N$ and $\sigma \pi \pi$ coupling constants. The mass is usually taken to be around $4 m_{\pi}$ to $6 m_{\pi}$.

\section{B. The $\Delta$ propagator}

There is an ambiguity as to the choice of propagator for a particle with spin-3/2, and a number of different propagators have been introduced 455 48]. One way of deriving a spin-3/2 propagator is to begin with the free Lagrangian for a massive spin-3/2 field [49. The propagator obtained from this Lagrangian has, in its most general form, a pole part and a non-pole part. The pole part is unique while the non-pole part depends on a complex parameter $A$. When the form of the $\pi N \Delta$ vertex is chosen correctly, the $S$-matrix and physical quantities are independent of $A$ [50]. Taking $A=-1$ gives the simplest form for the propagator, and is commonly called the Rarita-Schwinger (RS) propagator:

$$
P_{\mu \nu}(p)=\frac{\not p+m_{\Delta}}{p^{2}-m_{\Delta}^{2}+i \epsilon}\left[g_{\mu \nu}-\frac{1}{3} \gamma_{\mu} \gamma_{\nu}-\frac{1}{3 m_{\Delta}}\left(\gamma_{\mu} p_{\nu}-\gamma_{\nu} p_{\mu}\right)-\frac{2}{3 m_{\Delta}^{2}} p_{\mu} p_{\nu}\right]
$$


It is known that the RS propagator contains background or off-mass-shell spin-1/2 components, along with the spin-3/2 component (see, e.g. Ref. [51]). This becomes evident when the RS propagator is written in terms of spin projection operators, which are denoted by $\mathcal{P}_{i j}^{J}$, and are given by 52,51

$$
\begin{aligned}
\left(\mathcal{P}^{3 / 2}\right)_{\alpha \beta} & =g_{\alpha \beta}-\frac{1}{3} \gamma_{\alpha} \gamma_{\beta}-\frac{1}{3 p^{2}}\left(\not p \gamma_{\alpha} p_{\beta}+p_{\alpha} \gamma_{\beta} \not p\right) \\
\left(\mathcal{P}_{11}^{1 / 2}\right)_{\alpha \beta} & =\frac{1}{3} \gamma_{\alpha} \gamma_{\beta}-\frac{p_{\alpha} p_{\beta}}{p^{2}}+\frac{1}{3 p^{2}}\left(\not p \gamma_{\alpha} p_{\beta}+p_{\alpha} \gamma_{\beta} \not p\right) \\
\left(\mathcal{P}_{22}^{1 / 2}\right)_{\alpha \beta} & =\frac{p_{\alpha} p_{\beta}}{p^{2}} \\
\left(\mathcal{P}_{21}^{1 / 2}\right)_{\alpha \beta} & =\frac{1}{\sqrt{3} p^{2}}\left(p_{\alpha} p_{\beta}-\not p \gamma_{\alpha} p_{\beta}\right) \\
\left(\mathcal{P}_{12}^{1 / 2}\right)_{\alpha \beta} & =\frac{1}{\sqrt{3} p^{2}}\left(\not p p_{\alpha} \gamma_{\beta}-p_{\alpha} p_{\beta}\right)
\end{aligned}
$$

The RS propagator can then be written in terms of the spin projection operators defined above, as

$$
P_{\mu \nu}(p)=\frac{\not p+m_{\Delta}}{p^{2}-m_{\Delta}^{2}+i \epsilon} \mathcal{P}^{3 / 2}-\frac{2}{3 m_{\Delta}^{2}}\left(\not p+m_{\Delta}\right) \mathcal{P}_{22}^{1 / 2}+\frac{1}{\sqrt{3} m_{\Delta}}\left(\mathcal{P}_{12}^{1 / 2}+\mathcal{P}_{21}^{1 / 2}\right) .
$$

The spin-1/2 background could be considered as being unphysical, since the $\Delta(1232)$ is known experimentally to be a particle with spin-3/2.

Williams introduced a propagator proportional to the spin-3/2 projection operator 446,

$$
P_{\mu \nu}(p)=\frac{\not p+m_{\Delta}}{p^{2}-m_{\Delta}^{2}+i \epsilon} \mathcal{P}^{3 / 2}
$$

The Williams and RS propagators are identical when the $\Delta$ is on-mass-shell, since the spin$1 / 2$ components in the RS propagator are only present when the $\Delta$ is off-mass-shell. Also, when the Williams propagator is used there are no contributions to the $\pi N \leftarrow \pi N$ amplitude arising from the parts of the $\pi N \Delta$ vertices proportional to $x_{\Delta}$.

There have been attempts to fix $x_{\Delta}$ on theoretical grounds. Peccei [43] suggested that the choice $x_{\Delta}=-1 / 4$ ensures that there is no direct coupling to the spin- $1 / 2$ components of the RS propagator, but it was later shown [51] that the spin-1/2 components are always present, and cannot be removed by choosing a particular value of $x_{\Delta}$. Nath et al. 449] suggested that $x_{\Delta}=-1$ should be used if the $\pi N \Delta$ vertex is to be consistent with the principles of second quantization.

The $1 / p^{2}$ factor in the Williams propagator can cause numerical difficulties [7, 13]. Pascalutsa [47] used the Hamiltonian path-integral formulation to investigate the interacting spin-3/2 field and constructed a theory in which there is no coupling to the spin- $1 / 2$ components. For the case of the $s$ - and $u$-channel tree-level amplitudes for $\pi N$ scattering, the $\pi N \Delta$ vertex corresponds to the usual $\pi N \Delta$ vertex with $x_{\Delta}=0$, and the $\Delta$ propagator is the same as the Williams propagator but multiplied by $p^{2} / m_{\Delta}^{2}$. This extra factor of $p^{2}$ in the numerator fixes the problems caused by the $1 / p^{2}$ term in the spin-3/2 projection operator.

We consider two possibilities for the $\Delta$ propagator: (i) the Rarita-Schwinger propagator and general $\pi N \Delta$ vertex, with $x_{\Delta}$ as a free parameter, and (ii) the Pascalutsa propagator 
and vertex. In both cases the $\Delta$ is treated as a stable particle in the potential, and the width is generated dynamically, since the bare $s$-channel $\Delta$ pole diagram is dressed when the BS equation is solved.

\section{Form factors}

The integrals in Eqs. (5), (9), (10), and (14) are divergent, and so a regularization scheme must be implemented in order to obtain finite results. As is commonly done in meson-exchange models, we introduce form factors at each interaction vertex. These form factors represent the extended structure of the particles involved, and will ensure that all integrals are convergent by suppressing contributions at high momenta. Since at present it is not possible to calculate the appropriate form factors directly from QCD, phenomenological functions are usually chosen as the form factors, which have no connection to the underlying quark dynamics. A consequence of this is that free parameters, the cutoff masses that govern the range of suppression, are introduced into the model.

In meson-exchange models of the $N N$ interaction, the form factors depend only on the 4-momentum squared of the exchange particles. This cannot be done in $\pi N$ models because, for example, the form factors would provide no convergence for the $s$-channel pole diagrams. We therefore follow Pearce and Jennings [11], and make the assumption that the cutoff function associated with each vertex is a product of form factors that depend on the 4momentum squared of each particle present at the vertex. The $a b c$ vertex is therefore given by

$$
\Gamma_{a b c}=f_{a b c}\left(q_{a}^{2}, q_{b}^{2}, q_{c}^{2}\right) \mathcal{V}_{a b c}
$$

where $\mathcal{V}_{a b c}$ is the coupling operator obtained from the interaction Lagrangian, Eq. (15), and the associated form factor is of the separable form:

$$
f_{a b c}\left(q_{a}^{2}, q_{b}^{2}, q_{c}^{2}\right)=f_{a}\left(q_{a}^{2}\right) f_{b}\left(q_{b}^{2}\right) f_{c}\left(q_{c}^{2}\right)
$$

The 4-momenta squared of the legs of the vertices are denoted by $q_{a}^{2}, q_{b}^{2}$ and $q_{c}^{2}$. It is conventional to choose the normalization such that $f\left(m^{2}\right)=1$, where $m$ is the mass of the corresponding particle. Therefore, at the unphysical point when all three legs of a vertex are on-mass-shell, the corresponding product of form factors is equal to one.

The scalar functions $f\left(q^{2}\right)$ can essentially be chosen in an ad hoc manner, since very little is known about the off-mass-shell behaviour of the form factors. One possible choice is the multipole form factor:

$$
f_{\mathrm{I}}\left(q^{2}\right)=\left(\frac{\Lambda^{2}-m^{2}}{\Lambda^{2}-q^{2}}\right)^{n}
$$

where $\Lambda$ is the cutoff mass, and $n$ is an integer. Different forms of $f\left(q^{2}\right)$ have been used in the various models of $\pi N$ scattering. An example is [13,14

$$
f_{\mathrm{II}}\left(q^{2}\right)=\left(\frac{\left(\Lambda^{2}-m^{2}\right)^{2}}{\left(\Lambda^{2}-m^{2}\right)^{2}+\left(m^{2}-q^{2}\right)^{2}}\right)^{n} .
$$


The main difference between the two form factors $f_{\mathrm{I}}$ and $f_{\mathrm{II}}$ lies in the analytic structure in the complex $q_{0}$ plane (note $q^{2}=q_{0}^{2}-\mathbf{q}^{2}$ ). The function $f_{\mathrm{I}}$ has two poles along the real $q_{0}$ axis at

$$
q_{0}= \pm\left(\sqrt{\Lambda^{2}+\mathbf{q}^{2}}-i \epsilon\right)
$$

while $f_{\mathrm{II}}$ has four poles in the complex $q_{0}$ plane, located at

$$
q_{0}= \pm \sqrt{\Lambda^{2} \pm i\left(\Lambda^{2}-m^{2}\right)+\mathbf{q}^{2}}
$$

The form factors used by Pearce and Jennings [11] have a very similar structure to $f_{\mathrm{II}}$ in the complex $q_{0}$ plane. We note that $f_{\mathrm{II}}\left(q^{2}\right)$ always has poles in each of the four quadrants of the $q_{0}$ complex plane, irrespective of the value of $\Lambda$ (except when $\Lambda^{2}=m^{2}$ ). We should also mention that $f_{\mathrm{I}}\left(q^{2}\right)$ with $n=1$ could be considered as the propagator for a scalar particle with mass $\Lambda$, while $f_{\mathrm{II}}\left(q^{2}\right)$ with $n=1$ corresponds to the propagator for a resonance with mass $\Lambda$, and a width proportional to $\left(\Lambda^{2}-m^{2}\right)$. It has been shown that when a form factor has the form of a propagator for a resonance with a constant width, there is a violation of unitarity at all energies, which only becomes evident when a four-dimensional formulation is used [53].

As an example of the problems caused by this violation of unitarity, we can consider the dressed nucleon propagator. If the one-pion loop self-energy diagram is calculated using form factors similar to $f_{\mathrm{II}}\left(q^{2}\right)$ at each vertex, it turns out that the dressed nucleon has a width. This is of course unphysical, since the nucleon is well known to be stable particle. As a result, form factors with poles in the complex $q_{0}$ plane cannot be used to regularize loop diagrams in 4-D models. Therefore, here we use form factors of the form $f_{\mathrm{I}}$, in which case the unitarity violations do not occur.

\section{RENORMALIZATION}

As shown in Sec. [1], the bare vertices and propagators appearing in the $s$-channel pole diagrams in the potential become dressed when the potential is iterated in the BS equation. A renormalization procedure therefore must be carried out in order to fix the bare parameters such that the renormalized quantities are equal to their physical values. Since we include form factors at all vertices, the bare masses and coupling constants are finite.

\section{A. The dressed $\pi N N$ vertex}

The $\pi N N$ vertex renormalization constant $Z_{1 N}$ is defined in the usual way: the bare $\pi N N$ vertex differs from the dressed vertex by only a constant, which is $Z_{1 N}$, when sandwiched between Dirac spinors and all external legs are placed on-mass-shell. Therefore the vertex renormalization constant is defined by the relation

$$
\bar{u}(\mathbf{P}) \Gamma_{\pi N N}(q ; P) u(\mathbf{q})=Z_{1 N}^{-1} \bar{u}(\mathbf{P}) \Gamma_{\pi N N}^{(0)}(q ; P) u(\mathbf{q}),
$$

with $P^{2}=m_{N}^{2}$, and $q^{2}$ taken such that all three legs of the vertex are on-mass-shell. 


\section{B. The dressed nucleon propagator}

We now turn to the renormalization of the dressed nucleon propagator. Firstly, Lorentz invariance requires that the nucleon self-energy can be written as the sum of a vector and scalar part, i.e.

$$
\Sigma_{N}(P)=\not P A(s)+m_{N} B(s)
$$

where $A(s)$ and $B(s)$ are functions of $s$ only. In order to fix the bare nucleon mass, we require that the dressed propagator has a pole at the physical nucleon mass, i.e.

$$
\lim _{s \rightarrow m_{N}^{2}} d_{N}^{-1}(P)=0 .
$$

This requirement gives the following expression for the bare nucleon mass in terms of the functions $A$ and $B$ :

$$
m_{N}^{(0)}=m_{N}\left(1-A\left(m_{N}^{2}\right)-B\left(m_{N}^{2}\right)\right) .
$$

The residue of the dressed nucleon propagator at the nucleon pole is defined as the nucleon wave-function renormalization constant $Z_{2 N}$, i.e.

$$
\lim _{s \rightarrow m_{N}^{2}}\left(\not P-m_{N}\right) d_{N}(P)=Z_{2 N}
$$

which gives 31

$$
Z_{2 N}=\left[1-A\left(m_{N}^{2}\right)-2 m_{N}^{2}\left(A^{\prime}\left(m_{N}^{2}\right)+B^{\prime}\left(m_{N}^{2}\right)\right)\right]^{-1}
$$

where

$$
A^{\prime}\left(m_{N}^{2}\right)=\left.\frac{d}{d s} A(s)\right|_{s=m_{N}^{2}}, \quad B^{\prime}\left(m_{N}^{2}\right)=\left.\frac{d}{d s} B(s)\right|_{s=m_{N}^{2}} .
$$

The above expressions enable the renormalization constants to be calculated easily for the case of one-pion loop dressing, since it is not hard in this case to write $\Sigma_{N}(P)$ in terms of the functions $A(s)$ and $B(s)$. However, if the non-pole contributions to the nucleon self-energy are taken into account, then this would not be the simplest method of calculation. We introduce the scalar quantity $\Sigma_{N}^{\bar{u} u}(s)$, which is defined as the nucleon self-energy sandwiched between Dirac spinors, i.e.

$$
\begin{aligned}
\Sigma_{N}^{\bar{u} u}(s) & =\bar{u}(\mathbf{P}) \Sigma_{N}(P) u(\mathbf{P}) \\
& =\sqrt{s} A(s)+m_{N} B(s),
\end{aligned}
$$

since $\mathbf{P}=\mathbf{0}$ in the CM system. Taking note of Eqs. (34), (36), and (37), the renormalization constants $m_{N}^{(0)}$ and $Z_{2 N}$ can be written in terms of $\Sigma_{N}^{\bar{u} u}(s)$ as

$$
\begin{aligned}
& m_{N}^{(0)}=m_{N}-\Sigma_{N}^{\bar{u} u}\left(m_{N}^{2}\right), \\
& Z_{2 N}=\left[1-\left.2 m_{N} \frac{d}{d s} \Sigma_{N}^{\bar{u} u}(s)\right|_{s=m_{N}^{2}}\right]^{-1} .
\end{aligned}
$$

In this way we can include both the one-loop and the non-pole contributions to the mass shift and wavefunction renormalization $Z_{2 N}$ in a way consistent with the scattering formulation of the BS equation. 


\section{Renormalization of the BS equation}

In the $\pi N$ amplitude there should be factors of $\sqrt{Z_{2 N}}$ for each of the external nucleon legs, which result from the application of LSZ reduction [54] on the $\pi N \leftarrow \pi N$ Green's function. However, since we are not performing any explicit LSZ reduction, and we are assuming that the nucleon propagators in the kernel of the BS equation are bare propagators with physical masses, the factors of $\sqrt{Z_{2 N}}$ are not generated, and the $\pi N N$ and $\pi N \Delta$ couplings are effective coupling constants that should be set equal to the physical coupling constants. The only exception is with the $s$-channel pole diagrams. In this case, the solution of the BS equation generates new $s$-channel pole amplitudes in which the baryon propagators and $\pi N B$ vertices are dressed. This implies that bare masses and coupling constants should be used in the $s$-channel pole diagrams in the potential.

In order that the $\pi N$ amplitude has a pole at the physical nucleon mass, and that the residue at this pole is equal to the square of the physical $\pi N N$ coupling constant, the bare nucleon mass must be fixed by Eq. (39), and the bare $\pi N N$ coupling constant fixed using

$$
g_{\pi N N}^{R}=Z_{1 N}^{-1} \sqrt{Z_{2 N}} g_{\pi N N}^{(0)} .
$$

Here $g_{\pi N N}^{(0)}$ is the bare $\pi N N$ coupling constant, and $g_{\pi N N}^{R}$ is the renormalized coupling constant, which is set equal to the "experimental" $\pi N N$ coupling constant by fixing the value of $g_{\pi N N}^{(0)}$ correctly.

In principle, a similar renormalization procedure should be carried out for the $\Delta$. However, the pole in the $T$-matrix corresponding to the dressed $\Delta$ occurs in the complex $s$-plane, since the dressed $\Delta$ has a width. Therefore, in order to fix the bare $\pi N \Delta$ coupling constant, it would be necessary to analytically continue the BS equation into the complex $s$-plane [38. Rather than doing this, here both $m_{\Delta}^{(0)}$ and $f_{\pi N \Delta}^{(0)}$ are treated as free parameters. Since the $P_{33}$ partial wave is dominated by the $s$-channel $\Delta$ pole diagram, the bare $\Delta$ parameters are essentially fixed by the $P_{33}$ phase-shifts. The position at which the phase shifts go through $90^{\circ}$ is determined by the bare $\Delta$ mass, and the width of the resonance is related to the bare $\pi N \Delta$ coupling constant.

\section{SOLVING THE BS EQUATION}

To calculate quantities such as phase shifts and scattering lengths, we solve Eq. (5), i.e. the BS equation with the potential consisting the $s$ - and $u$-channel $N$ and $\Delta$ poles as well as $t$-channel $\rho$ and $\sigma$ exchange. In addition, the dressed $\pi N N$ vertex and nucleon self-energy need to be calculated so that the nucleon renormalization procedure can be performed.

\section{A. Partial wave expansion}

The nucleon propagator present in the $\pi N$ intermediate states can be separated into positive and negative energy components [55, which allows us to write $G_{\pi N}$ in terms of projection operators:

$$
G_{\pi N}(q ; P)=G^{\bar{u} u}\left(q_{0}, q ; s\right) \Lambda^{+}(\mathbf{q})-G^{\bar{v} v}\left(q_{0}, q ; s\right) \Lambda^{-}(-\mathbf{q})
$$


where $G^{\bar{u} u}$ and $G^{\bar{v} v}$ are given by

$$
\begin{aligned}
G^{\bar{u} u}\left(q_{0}, q ; s\right) & =\frac{m_{N}}{E_{q}} \frac{1}{\sqrt{s} / 2+q_{0}-E_{q}+i \epsilon} \frac{1}{\left(\sqrt{s} / 2-q_{0}\right)^{2}-\omega_{q}^{2}+i \epsilon}, \\
G^{\bar{v} v}\left(q_{0}, q ; s\right) & =\frac{m_{N}}{E_{q}} \frac{1}{\sqrt{s} / 2+q_{0}+E_{q}-i \epsilon} \frac{1}{\left(\sqrt{s} / 2-q_{0}\right)^{2}-\omega_{q}^{2}+i \epsilon},
\end{aligned}
$$

with $E_{q}=\sqrt{\mathbf{q}^{2}+m_{N}^{2}}$ and $\omega_{q}=\sqrt{\mathbf{q}^{2}+m_{\pi}^{2}}$. The positive and negative energy projection operators can be written in terms of Dirac spinors as

$$
\begin{aligned}
& \Lambda^{+}(\mathbf{q})=\sum_{r} u_{r}(\mathbf{q}) \bar{u}_{r}(\mathbf{q}), \\
& \Lambda^{-}(\mathbf{q})=-\sum_{r} v_{r}(\mathbf{q}) \bar{v}_{r}(\mathbf{q}) .
\end{aligned}
$$

The normalization of these spinors is defined by $u_{r}^{\dagger}(\mathbf{q}) u_{r}(\mathbf{q})=v_{r}^{\dagger}(\mathbf{q}) v_{r}(\mathbf{q})=1$.

The expansion given above in Eq. (42) is substituted into the BS equation. Multiplying the BS equation from the left and right by Dirac spinors yields a pair of coupled integral equations. If we introduce the notation (suppressing the Dirac indices)

$$
\begin{aligned}
& T^{\bar{u} u}\left(q^{\prime}, q ; P\right)=\bar{u}\left(\mathbf{q}^{\prime}\right) T\left(q^{\prime}, q ; P\right) u(\mathbf{q}), \\
& T^{\bar{v} u}\left(q^{\prime}, q ; P\right)=\bar{v}\left(-\mathbf{q}^{\prime}\right) T\left(q^{\prime}, q ; P\right) u(\mathbf{q}),
\end{aligned}
$$

and similarly for the potential, the BS equation can be written as two coupled equations for $T^{\bar{u} u}$ and $T^{\bar{v} u}$ :

$$
\begin{aligned}
T^{\bar{w} u}\left(q^{\prime}, q ; P\right)= & V^{\bar{w} u}\left(q^{\prime}, q ; P\right) \\
& -\frac{i}{(2 \pi)^{4}} \sum_{w^{\prime \prime}=u, v} \int d^{4} q^{\prime \prime} V^{\bar{w} w^{\prime \prime}}\left(q^{\prime}, q^{\prime \prime} ; P\right) G^{\bar{w}^{\prime \prime} w^{\prime \prime}}\left(q^{\prime \prime} ; P\right) T^{\bar{w}^{\prime \prime} u}\left(q^{\prime \prime}, q ; P\right),
\end{aligned}
$$

with $w=u, v$. There is a similar set of two coupled equations for the amplitudes $T^{\bar{u} v}$ and $T^{\bar{v} v}$, which are required along with $T^{\bar{u} u}$ and $T^{\bar{v} u}$ in the calculation of $m_{N}^{(0)}$ and $Z_{2 N}$.

We now reduce the number of dimensions from four to two, by removing the angular dependence using a partial wave expansion. Including spinor indices again, we can write each amplitude in the form

$$
A_{\lambda^{\prime} \lambda}^{\bar{w}^{\prime} w}\left(q^{\prime}, q ; P\right)=\chi_{\lambda^{\prime}}^{\dagger} \tilde{A}^{\bar{w}^{\prime} w}\left(q^{\prime}, q ; P\right) \chi_{\lambda}
$$

where $w$ and $w^{\prime}$ are either $u$ or $v$, and $\chi_{\lambda}$ is a Pauli spinor. The amplitude $\tilde{A}^{\bar{w}^{\prime} w}$ can be expanded in terms of partial wave amplitudes $A_{\ell \ell^{\prime} j}^{\bar{w}^{\prime} w}$ as

$$
\tilde{A}^{\bar{w}^{\prime} w}\left(q_{0}^{\prime}, \mathbf{q}^{\prime} ; q_{0}, \mathbf{q} ; s\right)=N\left(q^{\prime}, q\right) \sum_{\ell \ell^{\prime} j m} \mathcal{Y}_{\ell j m}(\hat{\mathbf{q}}) A_{\ell \ell^{\prime} j}^{\bar{w}^{\prime} w}\left(q_{0}^{\prime}, q^{\prime} ; q_{0}, q ; s\right) \mathcal{Y}_{\ell^{\prime} j m}^{\dagger}\left(\hat{\mathbf{q}}^{\prime}\right)
$$

where $q=|\mathbf{q}|, q^{\prime}=\left|\mathbf{q}^{\prime}\right|$, and $N\left(q^{\prime}, q\right)=-(2 \pi)^{4}\left(q^{\prime} q\right)^{-1}$. Also, the generalized Legendre polynomials are given by

$$
\mathcal{Y}_{\ell j m}(\hat{\mathbf{q}})=\sum_{m_{\ell} m_{s}}\left(\ell m_{\ell} \frac{1}{2} m_{s} \mid j m\right) Y_{\ell m_{\ell}}(\hat{\mathbf{q}}) \chi_{m_{s}}
$$


which are eigenstates of the magnitude of the total angular-momentum operator, $J^{2}$, its $z$-component, $J_{z}$, the magnitude of the orbital angular-momentum operator, $L^{2}$, and the magnitude of the spin operator, $S^{2}$. The partial wave amplitude can be written in terms of the original amplitude as

$$
A_{\ell \ell^{\prime} j}^{\bar{w}^{\prime} w}\left(q_{0}^{\prime}, q^{\prime} ; q_{0}, q ; s\right)=\frac{1}{N\left(q^{\prime}, q\right)} \int d \hat{\mathbf{q}}^{\prime} d \hat{\mathbf{q}}^{\mathcal{Y}_{\ell j m}^{\dagger}}\left(\hat{\mathbf{q}}^{\prime}\right) \tilde{A}^{\bar{w}^{\prime} w}\left(q_{0}^{\prime}, \mathbf{q}^{\prime} ; q_{0}, \mathbf{q} ; s\right) \mathcal{Y}_{\ell^{\prime} j m}(\hat{\mathbf{q}}) \text {. }
$$

Applying the partial wave decomposition to the BS equation and making use of the orthogonality of the generalized Legendre polynomials, we obtain

$$
\begin{aligned}
& T_{\ell j I}^{\bar{u} u}\left(q_{0}^{\prime}, q^{\prime} ; q_{0}, q ; s\right)=V_{\ell j I}^{\bar{u} u}\left(q_{0}^{\prime}, q^{\prime} ; q_{0}, q ; s\right) \\
& \quad+i \sum_{w^{\prime \prime}=u, v} \int_{-\infty}^{\infty} d q_{0}^{\prime \prime} \int_{0}^{\infty} d q^{\prime \prime} V_{\ell \ell^{\prime} j I}^{\bar{u} w^{\prime \prime}}\left(q_{0}^{\prime}, q^{\prime} ; q_{0}^{\prime \prime}, q^{\prime \prime} ; s\right) G^{\bar{w}^{\prime \prime} w^{\prime \prime}}\left(q_{0}^{\prime \prime}, q^{\prime \prime} ; s\right) T_{\ell^{\prime} \ell j I}^{\bar{w}^{\prime \prime} u}\left(q_{0}^{\prime \prime}, q^{\prime \prime} ; q_{0}, q ; s\right), \\
& T_{\ell^{\prime} \ell j I}^{\bar{v} u}\left(q_{0}^{\prime}, q^{\prime} ; q_{0}, q ; s\right)=V_{\ell^{\prime} \ell j I}^{\bar{v} u}\left(q_{0}^{\prime}, q^{\prime} ; q_{0}, q ; s\right) \\
& \quad+i \sum_{w^{\prime \prime}=u, v} \int_{-\infty}^{\infty} d q_{0}^{\prime \prime} \int_{0}^{\infty} d q^{\prime \prime} V_{\ell^{\prime} \ell j I}^{\bar{v} w^{\prime \prime}}\left(q_{0}^{\prime}, q^{\prime} ; q_{0}^{\prime \prime}, q^{\prime \prime} ; s\right) G^{\bar{w}^{\prime \prime} w^{\prime \prime}}\left(q_{0}^{\prime \prime}, q^{\prime \prime} ; s\right) T_{\ell^{\prime} \ell j I}^{\bar{w}^{\prime \prime} u}\left(q_{0}^{\prime \prime}, q^{\prime \prime} ; q_{0}, q ; s\right) .
\end{aligned}
$$

Note that the amplitude $A_{\ell \ell^{\prime} j}^{\bar{u} u}$ is diagonal in $\ell$, i.e. $\ell=\ell^{\prime}$, due to parity conservation, however amplitudes such as $A_{\ell^{\prime} j}^{\bar{u} v}$, which involve transitions between positive and negative energy nucleon states, are not diagonal in $\ell$. Each partial wave amplitude $T^{\bar{u} u}$ is labelled by the orbital angular momentum $\ell$, the total angular momentum $j$, where $j=\ell \pm \frac{1}{2}$, and the total isospin $I$, with $I=\frac{1}{2}$ or $I=\frac{3}{2}$.

\section{B. Analytic structure}

At this stage it is necessary to examine the analytic structure of the partial wave BS equations. We will carry out a Wick rotation [56], and analytically continue the $\pi N$ amplitude in the $q_{0}^{\prime}$ and $q_{0}^{\prime \prime}$ variables from the real axis to the imaginary axis. Before doing this, we must examine the singularity structure of the kernels of Eqs. (54) and (55) in the $q_{0}^{\prime \prime}$ plane, to make sure that there are no poles or cuts that could interfere with the Wick rotation. The residues of any poles present in the first and third quadrants need to be picked up, since we rotate the $q_{0}^{\prime \prime}$ integration contour from the real axis to the imaginary axis in an anti-clockwise direction. The presence of form factors in the potential ensures that the kernel is well behaved asymptotically, and as a result there is no contribution from the contour at infinity. There are three sources of analytic structure that we need to examine: (i) the $\pi N$ intermediate state, (ii) the potential, in which there are poles from the both the exchange particle propagators and the form factors, and (iii) the $\pi N T$-matrix itself.

The poles of the $\pi N$ two-body propagator $G^{\bar{u} u}\left(q_{0}^{\prime \prime}, q^{\prime \prime} ; s\right)$ in the complex $q_{0}^{\prime \prime}$ plane are located at

$$
\begin{aligned}
& q_{0}^{\prime \prime}=\omega_{N}^{+}\left(q^{\prime \prime}\right) \equiv-\sqrt{s} / 2+\sqrt{q^{\prime \prime 2}+m_{N}^{2}}-i \epsilon, \\
& q_{0}^{\prime \prime}=\omega_{\pi}^{ \pm}\left(q^{\prime \prime}\right) \equiv \sqrt{s} / 2 \mp\left(\sqrt{q^{\prime \prime 2}+m_{\pi}^{2}}-i \epsilon\right),
\end{aligned}
$$

corresponding to the positive energy nucleon pole, and the positive and negative energy pion poles respectively. $G^{\bar{v} v}\left(q_{0}^{\prime \prime}, q^{\prime \prime} ; s\right)$ has poles at $q_{0}^{\prime \prime}=\omega_{\pi}^{ \pm}$, and at 


$$
q_{0}^{\prime \prime}=\omega_{N}^{-}\left(q^{\prime \prime}\right) \equiv-\sqrt{s} / 2-\sqrt{q^{\prime \prime 2}+m_{N}^{2}}+i \epsilon
$$

which corresponds to the negative energy nucleon pole. If $\sqrt{s}>2 m_{\pi}$ it is possible for $\omega_{\pi}^{+}$to be in the first quadrant for $0<q^{\prime \prime}<q_{\max }^{\prime \prime}$, where $q_{\max }^{\prime \prime}=\sqrt{s / 4-m_{\pi}^{2}}$. The positive energy nucleon pole $\omega_{N}^{+}$can move into the third quadrant for $\sqrt{s}>2 m_{N}$. Therefore, if we stay below CM energies of $2 m_{N}$, we only need to pick up the residues from the positive energy pion propagator pole. The residues of $G^{\bar{u} u}\left(q_{0}^{\prime \prime}, q^{\prime \prime} ; s\right)$ and $G^{\bar{v} v}\left(q_{0}^{\prime \prime}, q^{\prime \prime} ; s\right)$ at $q_{0}^{\prime \prime}=\omega_{\pi}^{+}\left(q^{\prime \prime}\right)$ are

$$
\begin{aligned}
& G_{\mathrm{res}}^{\bar{u} u}\left(q^{\prime \prime} ; s\right)=-\frac{m_{N}}{2 E_{q^{\prime \prime}} \omega_{q^{\prime \prime}}} \frac{1}{\left(\sqrt{s}-E_{q^{\prime \prime}}-\omega_{q^{\prime \prime}}+i \epsilon\right)}, \\
& G_{\mathrm{res}}^{\bar{v} v}\left(q^{\prime \prime} ; s\right)=-\frac{m_{N}}{2 E_{q^{\prime \prime}} \omega_{q^{\prime \prime}}} \frac{1}{\left(\sqrt{s}+E_{q^{\prime \prime}}-\omega_{q^{\prime \prime}}\right)} .
\end{aligned}
$$

We now consider the singularities of all the diagrams present in the potential. Firstly, note that the partial wave potentials have the form

$$
\begin{aligned}
V_{\ell j I}^{\bar{u} u}\left(q_{0}^{\prime}, q^{\prime} ; q_{0}^{\prime \prime}, q^{\prime \prime} ; s\right) & =\frac{\pi}{N\left(q^{\prime}, q^{\prime \prime}\right)} \int_{-1}^{1} d x\left(P_{\ell}(x) f_{1}^{\bar{u} u}(s, t, u)+\frac{q^{\prime} q^{\prime \prime}}{\epsilon_{q^{\prime}} \epsilon_{q^{\prime \prime}}} P_{\ell \pm 1}(x) f_{2}^{\bar{u} u}(s, t, u)\right), \\
V_{\ell \ell^{\prime} j I}^{\bar{u} v}\left(q_{0}^{\prime}, q^{\prime} ; q_{0}^{\prime \prime}, q^{\prime \prime} ; s\right) & =\frac{\pi}{N\left(q^{\prime}, q^{\prime \prime}\right)} \int_{-1}^{1} d x\left(\frac{q^{\prime \prime}}{\epsilon_{q^{\prime \prime}}} P_{\ell}(x) f_{1}^{\bar{u} v}(s, t, u)+\frac{q^{\prime}}{\epsilon_{q^{\prime}}} P_{\ell^{\prime}}(x) f_{2}^{\bar{u} v}(s, t, u)\right),
\end{aligned}
$$

where $x=\hat{\mathbf{q}}^{\prime} \cdot \hat{\mathbf{q}}^{\prime \prime}$ and $\epsilon_{q}=E_{q}+m_{N}$. The forms of the partial wave potentials corresponding to $V^{\bar{v} v}$ and $V^{\bar{v} u}$ are very similar to Eqs. (61) and (62) respectively. For the $s$-channel baryon pole diagrams we have $f_{i}^{\bar{w} w} \propto 1 /\left(s-m_{B}^{2}\right)$ with $i=1,2$. Therefore, the only analytic structure in the $q_{0}^{\prime \prime}$ complex plane produced by the $s$-channel pole diagrams is due to the form factors on the external pion and nucleon legs, which we will look at shortly. However, for the $u$ - and $t$-channel diagrams we have $f_{i}^{\bar{w} w} \propto 1 /\left(z-m^{2}\right)$, where $z=u$ or $t$, and so the functions $f_{i}^{\bar{w} w}$ in this case depend on $q_{0}^{\prime \prime}$. After carrying out the $x$ integration in Eqs. (61) and (62), the partial wave potentials corresponding to the $u$ - and $t$-channel pole diagrams will involve terms such as

$$
\log \left(\frac{\left(q_{0}^{\prime}+\eta q_{0}^{\prime \prime}\right)^{2}-\left(q^{\prime}+\eta q^{\prime \prime}\right)^{2}-m^{2}+i \epsilon}{\left(q_{0}^{\prime}+\eta q_{0}^{\prime \prime}\right)^{2}-\left(q^{\prime}-\eta q^{\prime \prime}\right)^{2}-m^{2}+i \epsilon}\right),
$$

where $m$ is the mass of the particle, and $\eta=1(-1)$ for the $u$-channel ( $t$-channel) poles. Terms such as these generate logarithmic branch cuts in the $q_{0}^{\prime \prime}$ plane. For the $u$-channel pole diagrams, the branch points are at

$$
q_{0}^{\prime \prime}=-q_{0}^{\prime} \pm\left(\sqrt{\left(q^{\prime} \pm q^{\prime \prime}\right)^{2}+m_{B}^{2}}-i \epsilon\right)
$$

where $B=N$ or $\Delta$. The analytic structure for the $t$-channel exchange diagrams is very similar, namely there are branch points at

$$
q_{0}^{\prime \prime}=q_{0}^{\prime} \pm\left(\sqrt{\left(q^{\prime} \pm q^{\prime \prime}\right)^{2}+m_{A}^{2}}-i \epsilon\right)
$$

where $A=\rho$ or $\sigma$. 
Notice that in Eqs. (64) and (65) the positions of the branch points in the $q_{0}^{\prime \prime}$ plane depend on the external variable $q_{0}^{\prime}$, unlike the poles of the $\pi N$ intermediate state. Singularities that depend on $q_{0}^{\prime}$ are removed by the Wick rotation, since as well as rotating the $q_{0}^{\prime \prime}$ integration contour from the real to the imaginary axis, we analytically continue the $\pi N$ amplitude to the imaginary axis in $q_{0}^{\prime}$. The branch cuts move away from the integration contour as we rotate from the real to the imaginary axis, and in fact the branch points always stay a distance $m$ away from the integration contour, where $m$ is the mass of the exchange particle.

Next we consider the singularities of the form factors. The form factors corresponding to the external pion and nucleon legs have $n$-th order poles at

$$
\begin{aligned}
& q_{0}^{\prime \prime}=-\sqrt{s} / 2 \pm\left(\sqrt{q^{\prime \prime 2}+\Lambda_{N}^{2}}-i \epsilon\right), \\
& q_{0}^{\prime \prime}=\sqrt{s} / 2 \pm\left(\sqrt{q^{\prime \prime 2}+\Lambda_{\pi}^{2}}-i \epsilon\right),
\end{aligned}
$$

when the functional form given in Eq. (27) is used. The order of the poles depends on the choice for the form factor powers, i.e. $n_{\pi}$ and $n_{N}$. To guarantee that these form factor poles do not interfere with the Wick rotation, we require that they do not move into the first or third quadrants of the $q_{0}^{\prime \prime}$ plane. This means that we must have $\sqrt{s}<2 \Lambda_{\pi}$ and $\sqrt{s}<2 \Lambda_{N}$. Note that if form factors of the type given in Eq. (28) were being used, there would be poles from each form factor in both the first and third quadrants.

As a next step we look at the singularities in the form factors corresponding to the exchange particles. For the $u$-channel exchange diagrams there are branch points at

$$
q_{0}^{\prime \prime}=-q_{0}^{\prime} \pm\left(\sqrt{\left(q^{\prime} \pm q^{\prime \prime}\right)^{2}+\Lambda_{B}^{2}}-i \epsilon\right),
$$

where again $B=N$ or $\Delta$. Finally for the $t$-channel diagrams there are branch points at

$$
q_{0}^{\prime \prime}=q_{0}^{\prime} \pm\left(\sqrt{\left(q^{\prime} \pm q^{\prime \prime}\right)^{2}+\Lambda_{A}^{2}}-i \epsilon\right),
$$

where $A=\rho$ or $\sigma$. All of these branch points are removed by the Wick rotation, due to the $q_{0}^{\prime}$ dependence.

The remaining source of singularities which must be considered is the $T$-matrix, i.e. the solution of the BS equation. This can be done by looking at what happens as the potential is iterated in the BS equation. Singularities in the $q_{0}^{\prime}$ plane of $T\left(q^{\prime}, q ; P\right)$ are generated by pairs of poles pinching the $q_{0}^{\prime \prime}$ integration contour. This analytic structure therefore also occurs in the $q_{0}^{\prime \prime}$ plane of $T\left(q^{\prime \prime}, q ; P\right)$ which appears in the integrand of the BS equation. As the BS equation is iterated a hierarchy of branch cuts are generated. Higher order branch cuts arise from the pinching between lower order branch cuts and the singularities of the $\pi N$ intermediate state and potential. The positions of most of these cuts depends on $q_{0}^{\prime}$, and so do not cross the integration contour when both the $q_{0}^{\prime}$ and $q_{0}^{\prime \prime}$ axes are rotated. However, some of the higher order branch cuts do not depend on $q_{0}^{\prime}$ and so are not removed by a Wick rotation. If any pair of these cuts in the $q_{0}^{\prime \prime}$ plane protrude into both the first and third quadrants simultaneously, a simple Wick rotation becomes no longer possible.

Also, singularities pinching the $q_{0}^{\prime \prime}$ integration contour can produce cuts in the $\sqrt{s}$ plane, i.e. thresholds. If these thresholds are generated by singularities other than those from the 
form factors, they correspond to physical processes. The lowest-energy physical thresholds are at:

$$
\begin{aligned}
& \sqrt{s}=m_{N}+m_{\pi}, \\
& \sqrt{s}=m_{N}+2 m_{\pi}, \\
& \sqrt{s}=m_{\Delta}+2 m_{\pi}, \\
& \sqrt{s}=m_{N}+m_{\pi}+m_{\sigma} .
\end{aligned}
$$

However, if a cut in the $\sqrt{s}$-plane is generated by the pinching of a form factor pole and another singularity, the threshold is unphysical, since the cutoff mass does not correspond to the mass of a physical particle. Some examples of the unphysical thresholds generated by the BS equation include:

$$
\begin{aligned}
\sqrt{s} & =\Lambda_{N}+m_{\pi}, \\
\sqrt{s} & =m_{N}+\Lambda_{\pi}, \\
\sqrt{s} & =\Lambda_{N}+\Lambda_{\pi}, \\
\sqrt{s} & =m_{N}+m_{\pi}+\Lambda_{\sigma} .
\end{aligned}
$$

We therefore have to make sure that the cutoff masses are chosen to be large enough so that the unphysical thresholds occur above the highest CM energy for which we will solve the BS equation.

In summary, the BS equation can be solved for $\pi N$ scattering with our choice of form factors using a Wick rotation, provided that the cutoff masses are not too small. There are three conditions on the minimum values of the cutoff masses: (i) there are no form factor poles in the first or third quadrants of the $q_{0}^{\prime \prime}$ plane, (ii) any cuts produced by the pinching between form factor poles and other singularities are also not in the first or third quadrants, and finally (iii) all unphysical thresholds, which are generated by the form factors, are far away from the energy region in which we are interested. The minimum values for the cutoff masses that can be used in the BS equation are given in Table I. Here we have assumed that the two-pion production threshold is the maximum CM energy for which we will solve the BS equation.

If form factors of the type given in Eq. 28) are used, it is not possible to prevent the form factor singularities from interfering with the Wick rotation by making any appropriate choices for the cutoff masses. There will always be poles from the form factors in each quadrant of the $q_{0}^{\prime \prime}$ plane.

\section{Wick rotation}

Having looked at the analytic structure of the kernel of the partial wave BS equation, we are now in a position to perform a Wick rotation on Eqs. (54) and (55), by making the substitutions

$$
q_{0}^{\prime} \rightarrow i q_{0}^{\prime}, \quad q_{0}^{\prime \prime} \rightarrow i q_{0}^{\prime \prime},
$$

and picking up the residues from any poles in the first or third quadrants of the complex $q_{0}^{\prime \prime}$ plane. After Wick rotation, the partial wave BS equation becomes a system of four coupled integral equations. The first two equations for the half-off-shell $T$-matrix are 


$$
\begin{aligned}
& T_{\ell j I}^{\bar{u} u}\left(i q_{0}^{\prime}, q^{\prime} ; \bar{q}_{0}, \bar{q} ; s\right)=V_{\ell j I}^{\bar{u} u}\left(i q_{0}^{\prime}, q^{\prime} ; \bar{q}_{0}, \bar{q} ; s\right) \\
& \quad-\sum_{w^{\prime \prime}=u, v} \int_{-\infty}^{\infty} d q_{0}^{\prime \prime} \int_{0}^{\infty} d q^{\prime \prime} V_{\ell \ell^{\prime} j I}^{\bar{u} w^{\prime \prime}}\left(i q_{0}^{\prime}, q^{\prime} ; i q_{0}^{\prime \prime}, q^{\prime \prime} ; s\right) G^{\bar{w}^{\prime \prime} w^{\prime \prime}}\left(i q_{0}^{\prime \prime}, q^{\prime \prime} ; s\right) T_{\ell^{\prime} \ell j I}^{\bar{w}^{\prime \prime} u}\left(i q_{0}^{\prime \prime}, q^{\prime \prime} ; \bar{q}_{0}, \bar{q} ; s\right) \\
& \quad-\sum_{w^{\prime \prime}=u, v} \int_{0}^{q_{\max }^{\prime \prime}} d q^{\prime \prime} V_{\ell \ell^{\prime} j I}^{\bar{u} w^{\prime \prime}}\left(i q_{0}^{\prime}, q^{\prime} ; \omega_{\pi}^{+}\left(q^{\prime \prime}\right), q^{\prime \prime} ; s\right) G_{\mathrm{res}}^{\bar{w}^{\prime \prime} w^{\prime \prime}}\left(q^{\prime \prime} ; s\right) T_{\ell^{\prime} \ell j I}^{\bar{w}^{\prime \prime} u}\left(\omega_{\pi}^{+}\left(q^{\prime \prime}\right), q^{\prime \prime} ; \bar{q}_{0}, \bar{q} ; s\right), \\
& T_{\ell^{\prime} \ell j I}^{\bar{v} u}\left(i q_{0}^{\prime}, q^{\prime} ; \bar{q}_{0}, \bar{q} ; s\right)=V_{\ell^{\prime} \ell j I}^{\bar{v} u}\left(i q_{0}^{\prime}, q^{\prime} ; \bar{q}_{0}, \bar{q} ; s\right) \\
& \quad-\sum_{w^{\prime \prime}=u, v} \int_{-\infty}^{\infty} d q_{0}^{\prime \prime} \int_{0}^{\infty} d q^{\prime \prime} V_{\ell^{\prime} \ell j I}^{\bar{v} w^{\prime \prime}}\left(i q_{0}^{\prime}, q^{\prime} ; i q_{0}^{\prime \prime}, q^{\prime \prime} ; s\right) G^{\bar{w}^{\prime \prime} w^{\prime \prime}}\left(i q_{0}^{\prime \prime}, q^{\prime \prime} ; s\right) T_{\ell^{\prime} \ell j I}^{\bar{w}^{\prime \prime} u}\left(i q_{0}^{\prime \prime}, q^{\prime \prime} ; \bar{q}_{0}, \bar{q} ; s\right) \\
& \quad-\sum_{w^{\prime \prime}=u, v} \int_{0}^{q_{\max }^{\prime \prime}} d q^{\prime \prime} V_{\ell^{\prime} \ell j I}^{\bar{v} w^{\prime \prime}}\left(i q_{0}^{\prime}, q^{\prime} ; \omega_{\pi}^{+}\left(q^{\prime \prime}\right), q^{\prime \prime} ; s\right) G_{\mathrm{res}}^{\bar{w}^{\prime \prime} w^{\prime \prime}}\left(q^{\prime \prime} ; s\right) T_{\ell^{\prime} \ell j I}^{\bar{w}^{\prime \prime} u}\left(\omega_{\pi}^{+}\left(q^{\prime \prime}\right), q^{\prime \prime} ; \bar{q}_{0}, \bar{q} ; s\right) .
\end{aligned}
$$

In the above we have put the pion and nucleon in the initial state on-mass-shell. The on-shell relative momenta are denoted by $\bar{q}_{0}$ and $\bar{q}$, and are given by

$$
\bar{q}_{0}=\frac{1}{2}\left(\sqrt{\bar{q}^{2}+m_{N}^{2}}-\sqrt{\bar{q}^{2}+m_{\pi}^{2}}\right) \text {, }
$$

and

$$
\bar{q}=\sqrt{\frac{\left[s-\left(m_{N}+m_{\pi}\right)^{2}\right]\left[s-\left(m_{N}-m_{\pi}\right)^{2}\right]}{4 s}} .
$$

There are two additional equations (usually referred to as the "auxiliary equations") which are necessary in order to have a closed system of equations to solve: they are Eqs. (71) and (72) with $i q_{0}^{\prime}$ replaced with $\omega_{\pi}^{+}\left(q^{\prime}\right)$.

Finally, it is necessary to look at each term present in the potentials to check whether there are any remaining singularities after Wick rotation. For energies above the pion production threshold, the $u$-channel nucleon pole present in the potential in the one-dimensional parts of the auxiliary equations develops an imaginary part. This is due to a logarithmic singularity moving into the integration region, and must be handled carefully to ensure numerically stable results. We do this by carrying out a subtraction similar to Ref. [57]. There are no additional singularities caused by the form factors below the two-pion production threshold, provided the cutoff masses are chosen to be larger than the values given in Table 四.

Above $\sqrt{s}=2\left(m_{\sigma}+m_{\pi}\right)$ a cut in the $q_{0}^{\prime \prime}$ plane, generated by the pinching of the integration contour between the positive-energy pion pole and the $\sigma$ meson propagator, moves into the 1st quadrant. Therefore, above this value of the CM energy, it would become necessary to take this additional singularity into account when carrying out the Wick rotation. Here we consider CM energies below $\sqrt{s}=2\left(m_{\sigma}+m_{\pi}\right)$.

\section{Calculation of the phase shifts}

In order to determine the $\pi N$ phase shifts, the on-shell amplitude $T_{\ell j I}^{\bar{u} u}\left(\bar{q}_{0}, \bar{q} ; \bar{q}_{0}, \bar{q} ; s\right)$ needs to be calculated. This is done by analytic continuation of the half-off-shell amplitude to the 
on-shell point. In practice, the on-shell T-matrix is obtained using Eq. (71), with both the incoming and outgoing particles on-mass-shell.

The $\pi N$ two-body propagator $G_{\text {res }}^{\bar{u} u}\left(q^{\prime \prime} ; s\right)$ has a pole when the pion and nucleon are propagating on-shell, which occurs for $q^{\prime \prime}=\bar{q}$. This pole is related to the two-body unitarity cut. For energies above the $\pi N$ threshold we need to take care of this pole so as to obtain equations that can be solved numerically. We achieve this by writing $G_{\text {res }}^{\bar{u} u}\left(q^{\prime \prime} ; s\right)$ in terms of a principal-value part and an imaginary on-shell contribution, i.e.

$$
G_{\mathrm{res}}^{\bar{u} u}\left(q^{\prime \prime} ; s\right)=-\frac{m_{N}}{2 E_{q^{\prime \prime}} \omega_{q^{\prime \prime}}} \frac{\mathcal{P}}{\left(\sqrt{s}-E_{q^{\prime \prime}}-\omega_{q^{\prime \prime}}\right)}+\frac{i \pi m_{N}}{2 \bar{q} \sqrt{s}} \delta\left(q^{\prime \prime}-\bar{q}\right),
$$

where $\mathcal{P}$ denotes that the principal-value prescription should be used when the $q^{\prime \prime}$ integration is performed.

The $\pi N$ phase shifts $\delta_{\ell j I}$ and the inelasticities $\eta_{\ell j I}$ are obtained from the on-shell partial wave $T$-matrix using

$$
T_{\ell j I}^{\bar{u} u}\left(\bar{q}_{0}, \bar{q} ; \bar{q}_{0}, \bar{q} ; s\right)=-\frac{\bar{q} \sqrt{s}}{m_{N} \pi^{2}}\left(\frac{\eta_{\ell j I} e^{2 i \delta_{\ell j I}}-1}{2 i \bar{q}}\right) .
$$

The behaviour at threshold is more conveniently described in terms of scattering lengths and volumes, which are defined by the effective range expansion:

$$
\bar{q}^{2 \ell+1} \cot \delta_{\ell j I}=\frac{1}{a_{\ell j I}}+\frac{1}{2} r_{\ell j I} \bar{q}^{2}+\ldots
$$

Here $a_{\ell j I}$ is the scattering length and $r_{\ell j I}$ is the effective range.

\section{NUMERICAL RESULTS}

\section{A. Fits to the empirical $\pi N$ data}

We begin this section by listing the free parameters in our model. The five cutoff masses $\left(\Lambda_{N}, \Lambda_{\Delta}, \Lambda_{\pi}, \Lambda_{\rho}\right.$ and $\left.\Lambda_{\sigma}\right)$ are free, but are constrained to be larger than the minimum values given in Table $\mathbb{E}$. Due to the uncertainty in the values of the coupling constants other than $g_{\pi N N}$, we permit $f_{\pi N \Delta}, x_{\Delta}, g_{\rho}, \kappa_{\rho}$ and $g_{\sigma \pi \pi} g_{\sigma N N}$ to vary freely. The mass of the $\sigma$ meson, the bare $\Delta$ mass $m_{\Delta}^{(0)}$ and the bare $\pi N \Delta$ coupling constant $f_{\pi N \Delta}^{(0)}$ are also allowed to vary freely, although $m_{\Delta}^{(0)}$ and $f_{\pi N \Delta}^{(0)}$ are essentially fixed by the $P_{33}$ phase shifts. The bare nucleon mass $m_{N}^{(0)}$ and the bare $\pi N N$ coupling constant $g_{\pi N N}^{(0)}$ are not free parameters, but are determined by the renormalization procedure outlined in Section IV. We use the value $n_{a}=1$ for all the form factor powers, however it turns out that this choice is not crucial to the quality of the fit.

The free parameters are determined in $\chi^{2}$ fits to the $s$ - and $p$-wave single-energy phase shifts up to $360 \mathrm{MeV}$ pion laboratory energy, as well as the scattering lengths and volumes, from the VPI SM95 partial wave analysis [33. We carry out one fit using the RaritaSchwinger $\Delta$ propagator, and another using the Pascalutsa propagator. The coupling constants and particle masses for both fits are listed in Table $\amalg$. The resulting scattering lengths 
and volumes are given in Table [II, and the phase shifts are shown in Fig. 5. We see that the BS equation gives a good description of the $\pi N$ phase shifts. Notice that the results for the phase shifts in the $P_{11}$ partial wave are better when the Rarita-Schwinger $\Delta$ propagator is used.

As can be seen in Table [I], the values of $g_{\sigma \pi \pi} g_{\sigma N N}$ determined from both fits have negative signs, which means that the $\sigma$ contribution is repulsive in the $s$-waves and attractive in the $p$-waves, as was also found in Refs. [15, 17]. Note that we have used a low value for the $\pi N N$

coupling constant, i.e. $g_{\pi N N}^{2} / 4 \pi=13.5$. We have repeated the fits using $g_{\pi N N}^{2} / 4 \pi=14.3$ and found that the results are very similar to those shown in Fig. 5. Also, there are no significant differences between the coupling constants obtained from the fits using $g_{\pi N N}^{2} / 4 \pi=13.5$ and $g_{\pi N N}^{2} / 4 \pi=14.3$.

In Table [V] we compare our coupling constants to those extracted from $\pi N$ models based on the $K$-matrix approximation and 3-D reductions of the BS equation. Our coupling constants are in general consistent with those obtained from other models of $\pi N$ scattering. We note that $f_{\pi N \Delta}^{2} / 4 \pi$ is in the range 0.35 to 0.43 for all equations, except for the BS equation when the Pascalutsa $\Delta$ propagator is used, in which case the $\pi N \Delta$ coupling constant is about twice as large as the commonly accepted value. Our value of $x_{\Delta}$ is similar to those found in other models, and they are all in the range $-0.41<x_{\Delta}<-0.11$. All the models listed give similar values for $g_{\rho}^{2}$, and the values of $g_{\rho}^{2}$ all lie in the range $2.5<g_{\rho}^{2} / 4 \pi<3.36$. Our values are consistent with the KSRF relation [42] and the value found from the width of the pionic decay of the $\rho$ meson. There is a large range of values for $\kappa_{\rho}$, which vary between 1.44 and 6.6. Our value of $\kappa_{\rho}=2.66$ using the RS propagator is smaller than the VMD result of $\kappa_{\rho}=3.7$, and we get a value slightly larger than the VMD result $\left(\kappa_{\rho}=4.11\right)$ when we use the Pascalutsa $\Delta$ propagator. This suggests that within the uncertainty from the $\Delta$ propagator, we are consistent with vector meson dominance.

While we are to get a better fit to the $\pi N$ phase shifts using the Rarita-Schwinger $\Delta$ propagator than when using the Pascalutsa propagator, this does not necessarily suggest that the Rarita-Schwinger propagator is the correct spin-3/2 propagator. Other processes not included in the present model can give attractive contributions to the $P_{11}$ partial wave, such as the coupling to the $\pi \Delta$ channel or the inclusion of the $N^{*}(1440)$ resonance into the potential. More important is the observation that the different choices for the $\Delta$ propagator give rise to differences in the coupling constants. This highlights the importance of having a better understanding of how to construct propagators for higher-spin particles.

Having compared results using two different choices for the $\Delta$ propagator, hereafter we restrict ourselves to the Rarita-Schwinger propagator.

\section{B. Contributions to the phase shifts}

In Fig. 6 we show how the total phase shifts are built up from the contributions of the individual Feynman diagrams in the potential. The $u$-channel nucleon pole is strongest in the $S_{31}$ and $P_{33}$ partial waves, but also gives important contributions to $P_{13}$ and $P_{31}$. The $s$ channel nucleon pole generates the repulsion in the $P_{11}$ phase shifts, and gives a very small contribution to $S_{11}$. The $u$-channel $\Delta$ diagram plays a very important role in all partial waves except $S_{31}$ and $P_{33}$, as it gives a large repulsive contribution to $S_{11}$, and gives strong attractive contributions to $P_{11}, P_{13}$ and $P_{31}$. The $s$-channel $\Delta$ pole diagram dominates the 
$P_{33}$ phase shifts, but also gives an important contribution to the $S_{31}$ partial wave, and a tiny contribution to $P_{31}$. The contributions from the $s$-channel $\Delta$ pole to $S_{31}$ and $P_{31}$ result from the spin-1/2 components of the Rarita-Schwinger propagator. The $\rho$ exchange contributions are largest in the $s$-waves, but are also significant in the $p$-waves except for $P_{33}$. Likewise, $\sigma$ exchange is stronger in the $s$-waves than the $p$-waves, although the $\sigma$ exchange contributions are quite small in all partial waves.

The $S_{11}$ phase shifts are dominated by $\rho$ exchange and the $u$-channel $\Delta$ pole. By itself, the attraction generated by $\rho$ exchange is far too strong, but is partially cancelled by the repulsive $u$-channel $\Delta$ pole. Except for the $u$-channel nucleon pole, all of the diagrams contributing to the $S_{31}$ phase shifts are repulsive. The largest contributions to this repulsion come from $\rho$ exchange and the spin- $1 / 2$ components of the $s$-channel $\Delta$ pole.

The $s$-channel nucleon pole causes the $P_{11}$ phase shifts to be negative at low energies. The attraction that causes the phase shifts to change sign is dominated by the $u$-channel $\Delta$ pole and the $\rho$ exchange diagram.

The $u$-channel nucleon pole is strong and repulsive in the $P_{13}$ and $P_{31}$ partial waves. In fact, by itself, the $u$-channel nucleon pole almost gives the correct $P_{31}$ phase shifts. However, the $\rho$ exchange diagram gives a large repulsive contribution to $P_{31}$, causing the phase shifts to deviate strongly from the phase shift analysis. The attraction provided by the $u$-channel $\Delta$ pole to $P_{31}$ almost cancels the $\rho$ contribution completely. Similarly, the $P_{13}$ phase shifts are far too repulsive without the strong attraction produced by the $u$-channel $\Delta$ pole.

The $P_{33}$ phase shifts are of course dominated by the $s$-channel $\Delta$ pole, with the background contribution primarily coming from the $u$-channel nucleon pole.

\section{Dressing of the $\pi N N$ vertex}

As can be seen in Table II], the cutoff masses we obtain turn out to be quite large. This results in the dressing being very significant, as is evident from the large size of the bare $N$ and $\Delta$ masses. In view of the significance of the dressing, it is interesting to examine the effect of dressing on the $\pi N N$ form factor. When both nucleons in the bare $\pi N N$ vertex are placed on-mass-shell, the bare $\pi N N$ vertex only involves the pion form factor, and is given by

$$
\Gamma_{\pi N N}^{(0) \bar{u} u}\left(q_{0}, \mathbf{q} ; s\right)=f_{\pi}\left(q_{\pi}^{2}\right) \mathcal{V}_{\pi N N}^{\bar{u} u}\left(q_{0}, \mathbf{q} ; s\right)
$$

where $s=m_{N}^{2}$, and $q_{\pi}^{2}$ is the 4-momentum squared of the pion. The bare pion form factor is

$$
f_{\pi}\left(q_{\pi}^{2}\right)=\left(\frac{\Lambda_{\pi}^{2}-m_{\pi}^{2}}{\Lambda_{\pi}^{2}-q_{\pi}^{2}}\right)^{n_{\pi}}
$$

and we take $n_{\pi}=1$. The renormalized pion form factor can then be introduced as

$$
f_{\pi}^{R}\left(q_{\pi}^{2}\right)=\left.Z_{1} \frac{\Gamma_{\pi N N}^{\bar{u} u}\left(q_{0}, q ; s\right)}{\mathcal{V}_{\pi N N}^{\bar{u} u}\left(q_{0}, q ; s\right)}\right|_{P_{11}},
$$

which has the property that $f_{\pi}^{R}\left(m_{\pi}^{2}\right)=1$, and where $q_{0}$ and $q$ are related to the pion 4-momentum squared by 


$$
\begin{aligned}
q_{0} & =\frac{1}{2 m_{N}}\left(m_{N}^{2}-q_{\pi}^{2}\right), \\
q & =\frac{1}{2} \sqrt{q_{\pi}^{2}\left(\frac{q_{\pi}^{2}}{m_{N}^{2}}-4\right)} .
\end{aligned}
$$

In Eq. (80) we have taken the $P_{11}$ partial wave of the quantities $\Gamma_{\pi N N}^{\bar{u} u}\left(q_{0}, \mathbf{q} ; s\right)$ and $\mathcal{V}_{\pi N N}^{\bar{u} u}\left(q_{0}, \mathbf{q} ; s\right)$. By comparing the slope of the function $f_{\pi}^{R}\left(q_{\pi}^{2}\right)$ at $q_{\pi}^{2}=0$ with a monopole form factor with cutoff mass $\Lambda_{\pi}^{R}$, we can obtain a value for the renormalized pion cutoff mass. We find that $\Lambda_{\pi}^{R}=1.22 \mathrm{GeV}$, which is softer than the bare pion form factor (recall that $\Lambda_{\pi}=1.77 \mathrm{GeV}$ ). This is consistent with previous calculations [59 61], which have found that dressed form factors are softer than the corresponding bare form factors.

We can introduce the quantity $\Delta_{\pi}$ as a measure of the variation between the renormalized pion form factor at $q_{\pi}^{2}=m_{\pi}^{2}$ and $q_{\pi}^{2}=0$, i.e.

$$
\Delta_{\pi}=1-f_{\pi}^{R}(0) .
$$

We find that $\Delta_{\pi}=1.3 \%$, which indicates that our dressed $\pi N N$ form factor is a very slowlyvarying function of the pion mass. Our value of $\Delta_{\pi}$ is somewhat smaller than the value of $3 \%$ obtained using other methods 62.

\section{Different choice of form factors}

With the choice of form factors we have used so far (hereafter referred to as model I), the effect of dressing is significant. We now consider the case where there is a form factor only on the pion (referred to as model II), which is arrived at from the parameterization of form factors used in model I by taking the limit $\Lambda_{h} \rightarrow \infty$ for $h=N, \Delta, \rho$, and $\sigma$. With this choice of form factor there is only one cutoff mass, rather than five, and so the number of free parameters is reduced by four. All intermediate states contain the pion propagator, and therefore a cutoff function still appears in all loop diagrams to provide convergence. In model II the pion form factor is used to vary the off-mass-shell behaviour of the pion. This in principle could be constrained by the soft-pion theorems.

In Table $\nabla$ we show three sets of parameters obtained from fits to the on-shell $\pi N$ data, corresponding to the choices $n_{\pi}=2, n_{\pi}=4$, and $n_{\pi}=10$. The resulting phase shifts are all of the same quality as the results using the Rarita-Schwinger $\Delta$ propagator shown in Fig. 5 . The coupling constants resulting from the three model II fits are similar to the coupling constants obtained using model I, although $\kappa_{\rho}$ is smaller in model II than in model I, and

$g_{\sigma \pi \pi} g_{\sigma N N}$ has a positive sign, whereas in model I it is negative. As with model I, there are no significant changes to the quality of the fits or values of the coupling constants obtained when the fits are repeated using $g_{\pi N N}^{2} / 4 \pi=14.3$ as the physical $\pi N N$ coupling constant.

The main difference between the two models is that in model II the effect of dressing is not as significant as in model I. The bare baryon masses are much closer to the physical masses. The renormalized pion cutoff masses and values of $\Delta_{\pi}$ for model II are given in Table VI. We see that the values of $\Lambda_{\pi}^{R}$ are smaller than in model I, and are close to the value of $\Lambda_{\pi}^{R} \approx 0.8 \mathrm{GeV}$ advocated by some authors [63]. The values of $\Delta_{\pi}$ are consistent with previous calculations [62] of the difference between $f_{\pi}^{R}\left(m_{\pi}^{2}\right)$ and $f_{\pi}^{R}(0)$. 


\section{E. Above the $2 \pi$ production threshold}

In order to see what happens at energies above the $2 \pi$ production threshold, we show the phase shifts up to $600 \mathrm{MeV}$ pion laboratory energy in Fig. 7. The results from model II were obtained using $n_{\pi}=4$. In the $P_{11}$ and $P_{33}$ partial waves both models give almost identical results, but there are some differences between models I and II in the higher energy region in the other partial waves.

Both the $P_{13}$ and $P_{31}$ phase shifts are quite good over the full range of energies, which is a reflection of the fact that below $600 \mathrm{MeV}$ there are no resonances in these partial waves and the inelasticity is negligible. We require a little more attraction at the higher energies in the $S_{31}$ and $P_{33}$ partial waves, while a significant amount of additional attraction is required for $S_{11}$ above $300 \mathrm{MeV}$, and for $P_{11}$ above around $450 \mathrm{MeV}$.

It is not unexpected that there are some discrepancies in the higher energy region. Here the $S_{11}$ and $P_{11}$ partial waves exhibit resonance behaviour not included in the present model. There are a number of modifications that could be made to our model in order to improve the agreement with experiment for this larger energy range. Firstly, it may be necessary for three-body unitarity to be satisfied. Amongst other things, this will involve replacing the nucleon propagator in the $\pi N$ intermediate states with a dressed propagator. Extending the model to include the coupling to inelastic channels and the possible addition of explicit nucleon resonances into the potential will be essential at energies above $360 \mathrm{MeV}$. To fully understand the $\pi N$ amplitude at these energies, the coupling to inelastic channels must first be included, and if the fit to the phase shifts is still unsatisfactory, explicit bare $N^{*}$ baryon poles may need to be included in the potential.

The $S_{11}$ partial wave would be improved by the inclusion of the coupling to the $\eta N$ channel, and also $S_{11}$ resonances such as the $N^{*}(1535)$ and $N^{*}(1650)$ may need to be included in the potential. The coupling to the $\pi \Delta$ and $\sigma N$ channels and possibly the inclusion of the $N^{*}(1440)$ resonance into the potential would be necessary to improve the $P_{11}$ phase shifts above $450 \mathrm{MeV}$.

\section{CONCLUSION}

In this work we have presented a description of pion-nucleon scattering based on the four-dimensional Bethe-Salpeter equation. The kernel of the equation is based on a chiral Lagrangian that includes in addition to pions and nucleons, the $\Delta(1232)$ and the $\rho$ and $\sigma$ mesons. The potential obtained from this Lagrangian consists of $s$ - and $u$-channel $N$ and $\Delta$ pole diagrams as well as $t$-channel $\rho$ and $\sigma$ exchanges. Convergence of all integrals is guaranteed by the use of cutoff fuctions associated with each vertex. Two different parameterizations of the cutoff functions were considered: in model I the cutoff function was taken to be a product of form factors depending on the four-momentum squared of each particle

present at the vertex. In model II the cutoff function was taken to depend only on the pion four-momentum squared. The parameters of the potential were adjusted to fit the empirical $s$ - and $p$-wave phase shifts up to a pion laboratory kinetic energy of $360 \mathrm{MeV}$. Both models give good fits to the $\pi N$ scattering data, and the resulting coupling constants are consistent with the commonly accepted values extracted from other observables. 
While most of our results were for the Rarita-Schwinger $\Delta$ propagator, we compared the results of fits performed using the Rarita-Schwinger and Pascalutsa propagators for the case of the model I form factors. The differences in the coupling constants obtained suggests that a complete understanding of the baryon resonances with higher-spin is not possible without having unique higher-spin propagators.

The good fits to the on-shell $\pi N$ data for energies below $360 \mathrm{MeV}$ suggests that a model of the $\pi N$ interaction based on the Bethe-Salpeter equation could form the basis for the analysis of pion photoproduction by the proper $U(1)$ gauging of the Lagrangian [31], and the analysis of the baryon resonances near and above the threshold for pion production. In fact, by extending the calculations to pion energies up to $600 \mathrm{MeV}$, we observe that in partial waves that don't have large inelasticities and don't exhibit evidence of baryon states, both our models give good representations of the data. For partial waves with large inelasticity there is also evidence for baryon resonances, and as a result we need to include first the coupling to inelastic channels, and then include bare baryon states into the potential if necessary.

Although we have not included full three-body unitarity, which would require the dressing of the nucleon propagators and $\pi N N$ vertices, we have included those contributions to threebody unitarity resulting from the fact that we have not carried out any three-dimensional reduction, and as such our potentials depend on the relative energy. The inclusion of the dressed nucleon in the $\pi N$ intermediate states will increase the number of coupled channels, and is under investigation.

By calculating off-mass-shell $\pi N$ amplitudes using the Bethe-Salpeter equation we can examine the low energy theorems, and study the questions associated with the analytic continuation of the physical $\pi N$ data to the Cheng-Dashen point [64, and the changes in the $\pi N$ sigma term as one goes from the Weinberg point, where the four-momenta of the pions is zero, to the Cheng-Dashen point, where the pions are on-mass-shell. This question is presently being examined and could shed some light on the inconsistencies between the "observed" $\Sigma_{N}$ and $\sigma_{N}$ as extracted from QCD models.

It is clear from the work presented here that constraints on the coupling constants and form factors need to be improved before it can be established whether or not the present potential includes all the physics of $\pi N$ scattering at low energies. We are of the opinion that such constraints, particularly on the coupling constants, should come from QCD or QCD-based models.

\section{ACKNOWLEDGMENTS}

This work was conducted while A. D. L. held an Australian Postgraduate Award. We acknowledge the financial support of the Australian Research Council. We are also indebted to the South Australian Centre for Parallel Computing for access to their computing facilities.

[1] J. Fröhlich, K. Schwarz, L. Streit and H. F. K. Zingl, Phys. Rev. C 25, 2591 (1982). 
[2] R. J. McLeod and I. R. Afnan, Phys. Rev. C 32, 222 (1985).

[3] S. Nozawa, B. Blankleider, and T.-S.H. Lee, Nucl. Phys. A513, 459 (1990).

[4] M. G. Fuda, Phys. Rev. C 52, 2875 (1995).

[5] Y. Elmessiri and M. G. Fuda, Phys. Rev. C 57, 2149 (1998).

[6] M. G. Olsson and E. T. Osypowski, Nucl. Phys. B101, 136 (1975).

[7] D. Bofinger and W. S. Woolcock, Nuovo Cim. 104A, 1489 (1991).

[8] P. F. A. Goudsmit, H. J. Leisi, E. Matsinos, B. L. Birbrair and A. B. Gridnev, Nucl. Phys. A575, 673 (1994).

[9] T. Feuster and U. Mosel, Phys. Rev. C 58, 457 (1998).

[10] A. Yu. Korchin, O. Scholten, and R. G. E. Timmermans, Phys. Lett. B 438, 1 (1998).

[11] B. C. Pearce and B. K. Jennings, Nucl. Phys. A528, 655 (1991).

[12] C. Lee, S. N. Yang and T.-S. H. Lee, J. Phys. G 17, L131 (1991).

[13] F. Gross and Y. Surya, Phys. Rev. C 47, 703 (1993).

[14] C. T. Hung, S. N. Yang and T.-S. H. Lee, J. Phys. G 20, 1531 (1994).

[15] C. Schütz, J. W. Durso, K. Holinde and J. Speth, Phys. Rev. C 49, 2671 (1994).

[16] C. Schütz, J. Haidenbauer, J. Speth and J. W. Durso, Phys. Rev. C 57, 1464 (1998).

[17] V. Pascalutsa and J. A. Tjon, Nucl. Phys. A631, 534c (1998); Phys. Lett. B 435, 245 (1998).

[18] V. Pascalutsa, Ph.D. thesis, University of Utrecht, 1998.

[19] E. E. Salpeter and H. A. Bethe, Phys. Rev. 84, 1232 (1951).

[20] L. Jäde, Phys. Rev. C 58, 96 (1998).

[21] T. Sato and T.-S. H. Lee, Phys. Rev. C 54, 2660 (1996).

[22] P. J. Ellis and H.-B. Tang, Phys. Rev. C 57, 3356 (1998).

[23] E. A. Veit, B. K. Jennings, and A. W. Thomas, Phys. Rev. D 33, 1859 (1986).

[24] B. C. Pearce and I. R. Afnan, Phys. Rev. C 34, 991 (1986).

[25] D. Lu, S. C. Phatak, and R. H. Landau, Phys. Rev. C 51, 2207 (1995).

[26] F. Gross, Phys. Rev. C 26, 2203 (1982).

[27] E. D. Cooper, B. K. Jennings, P. A. M. Guichon, and A. W. Thomas, Nucl. Phys. A469, 717 (1987).

[28] R. Yaes, Phys. Rev. D 3, 3086 (1971).

[29] E. D. Cooper and B. K. Jennings, Nucl. Phys. A483, 601 (1988).

[30] A. D. Lahiff and I. R. Afnan, Phys. Rev. C 56, 2387 (1997).

[31] C. H. M. van Antwerpen and I. R. Afnan, Phys. Rev. C 52, 554 (1995).

[32] H. M. Nieland and J. A. Tjon, Phys. Lett. B 27B, 309 (1968); J. A. Tjon and H. M. Nieland, in The Padé approximant in theoretical physics, eds. G. Baker Jr. and J. L. Gammel, (Academic Press, 1970), p. 289; H. M. Nieland, Ph.D. thesis, University of Nijmegen, 1971.

[33] R. A. Arndt, I. I. Strakovsky, R. L. Workman, and M. M. Pavan, Phys. Rev. C 52, 2120 (1995).

[34] R. W. Haymaker, Phys. Rev. 181, 2040 (1969).

[35] T. E. O. Ericson, B. Loiseau, J. Rahm, J. Blomgren, N. Olsson, and A. W. Thomas, hep-ph/9811515.

[36] M. C. M. Rentmeester, R. A. M. Klomp, and J. J. de Swart, Phys. Rev. Lett. 81, 5253 (1998).

[37] J. J. de Swart, M. C. M. Rentmeester, and R. G. E. Timmermans, nucl-th/9802084

[38] J. A. Elsey and I. R. Afnan, Phys. Rev. D 40, 2353 (1989).

[39] G. E. Brown and W. Weise, Phys. Rep. 22, 279 (1975).

[40] J. A. Niskanen, Phys. Lett. B 107B, 344 (1981). 
[41] J. J. Sakurai, Phys. Rev. Lett. 17, 1021 (1966).

[42] K. Kawarabayashi and M. Suzuki, Phys. Rev. Lett. 16, 255 (1966); Riazuddin and Fayyazuddin, Phys. Rev. 147, 1071 (1966).

[43] R. D. Peccei, Phys. Rev. 176, 1812 (1968).

[44] G. Höhler and E. Pietarinen, Nucl. Phys. B95, 210 (1975).

[45] W. Rarita and J. Schwinger, Phys. Rev. 60, 61 (1941).

[46] H. T. Williams, Phys. Rev. C 31, 2297 (1985).

[47] V. Pascalutsa, Phys. Rev. D 58, 096002 (1998).

[48] H. Haberzettl, nucl-th/9812043.

[49] L. M. Nath, B. Etemadi and J. D. Kimel, Phys. Rev. D 3, 2153 (1971); J. D. Kimel and L. M. Nath, Phys. Rev. D 6, 2132 (1972).

[50] S. Kamefuchi, L. O'Raifeartaigh and A. Salam, Nucl. Phys. 28, 529 (1961).

[51] M. Benmerrouche, R. M. Davidson and N. C. Mukhopadhyay, Phys. Rev. C 39, 2339 (1989).

[52] P. van Nieuwenhuizen, Phys. Rep. 68, 189 (1981).

[53] G. Ramalho, A. Arriaga, and M. T. Peña, nucl-th/9808060.

[54] H. Lehmann, K. Symanzik, and W. Zimmerman, Nuovo Cim. 1, 205 (1955).

[55] H. Garcilazo, Phys. Rev. C 47, 957 (1993).

[56] G. C. Wick, Phys. Rev. 96, 1124 (1954).

[57] F. Gross, J. W. van Orden, and K. Holinde, Phys. Rev. C 45, 2094 (1992).

[58] R. Koch and E. Pietarinen, Nucl. Phys. A336, 331 (1980).

[59] B. C. Pearce, in 14th International IUPAP Conference on Few-Body Problems in Physics, Contributed papers, edited by F. Gross (College of William \& Mary, Williamsburg, VA, 1994), p. 294.

[60] L. C. Liu, Q. Haider, and J. T. Londergan, Phys. Rev. C 51, 3427 (1995).

[61] S. Kondratyuk, and O. Scholten, Phys. Rev. C 59, 1070 (1999).

[62] S. A. Coon and M. D. Scadron, Phys. Rev. C 23, 1150 (1981); ibid. 42, 2256 (1990);

[63] A. W. Thomas, S. Théberge, and G. A. Miller, Phys. Rev. D 22, 1980 (2838); A. W. Thomas and K. Holinde, Phys. Rev. Lett. 63, 2025 (1989).

[64] T. P. Cheng and R. Dashen, Phys. Rev. Lett. 26, 594 (1971). 


\begin{tabular}{lr}
\hline \hline cutoff mass & minimum value \\
\hline$\Lambda_{N}$ & $m_{N}+3 m_{\pi}$ \\
$\Lambda_{\Delta}$ & $m_{N}+3 m_{\pi}$ \\
$\Lambda_{\pi}$ & $\left(m_{N}+3 m_{\pi}\right) / 2$ \\
$\Lambda_{\rho}$ & $\left(m_{N}+m_{\pi}\right) / 2$ \\
$\Lambda_{\sigma}$ & $\left(m_{N}+m_{\pi}\right) / 2$ \\
\hline \hline
\end{tabular}

TABLE I. The minimum allowed values of the cutoff masses in terms of the nucleon and pion masses.

\begin{tabular}{lrr}
\hline \hline coupling constants & RS & Pas \\
\hline$g_{\pi N N}^{2} / 4 \pi$ & 13.5 & 13.5 \\
$g_{\pi N N}^{(0) 2} / 4 \pi$ & 1.80 & 12.1 \\
$f_{\pi N \Delta}^{2} / 4 \pi$ & $\mathbf{0 . 3 6 5}$ & $\mathbf{0 . 7 4 1}$ \\
$f_{\pi N \Delta}^{(0) 2} / 4 \pi$ & $\mathbf{0 . 3 7}$ & $\mathbf{0 . 1 9 3}$ \\
$x_{\Delta}$ & $\mathbf{- 0 . 1 1}$ & - \\
$g_{\rho}^{2} / 4 \pi$ & $\mathbf{2 . 8 8}$ & $\mathbf{2 . 7 3}$ \\
$\kappa_{\rho}$ & $\mathbf{2 . 6 6}$ & $\mathbf{4 . 1 1}$ \\
$g_{\sigma \pi \pi} g_{\sigma N N} / 4 \pi$ & $\mathbf{- 0 . 4 1}$ & $\mathbf{3 . 8 0}$ \\
\hline$m_{S s e s}$ & & 0.939 \\
\hline$m_{N}$ & 0.939 & 1.72 \\
$m_{N}^{(0)}$ & 1.34 & 1.232 \\
$m_{\Delta}$ & 1.232 & $\mathbf{2 . 6 0}$ \\
$m_{\Delta}^{(0)}$ & $\mathbf{2 . 3 0 5}$ & 0.138 \\
$m_{\pi}$ & 0.138 & 0.769 \\
$m_{\rho}$ & 0.769 & $\mathbf{0 . 6 9}$ \\
$m_{\sigma}$ & $\mathbf{0 . 6 5}$ & $\mathbf{4 . 9 0}$ \\
\hline$\Lambda_{N}$ & $\mathbf{3 . 1 7}$ & $\mathbf{3 . 2 0}$ \\
$\Lambda_{\Delta}$ & $\mathbf{4 . 5 6}$ & $\mathbf{1 . 7 6}$ \\
$\Lambda_{\pi}$ & $\mathbf{1 . 7 7}$ & $\mathbf{3 . 0 6}$ \\
$\Lambda_{\rho}$ & $\mathbf{3 . 6 7}$ & $\mathbf{4 . 2 6}$ \\
$\Lambda_{\sigma}$ & $\mathbf{1 . 3 0}$ & \\
\hline \hline & & \\
\hline
\end{tabular}

TABLE II. The coupling constants and particle masses obtained using the Rarita-Schwinger (RS) and Pascalutsa (Pas) $\Delta$ propagators. The quantities in boldface were varied in the fits. All masses are in $\mathrm{GeV}$. 


\begin{tabular}{lrrrr}
\hline \hline$\ell_{2 I 2 j}$ & BS (RS) & BS (Pas) & SM95 & KH80 \\
\hline$S_{11}$ & 0.177 & 0.172 & 0.175 & 0.173 \\
$S_{31}$ & -0.101 & -0.105 & -0.087 & -0.101 \\
$P_{11}$ & -0.083 & -0.058 & -0.068 & -0.081 \\
$P_{13}$ & -0.032 & -0.031 & -0.022 & -0.030 \\
$P_{31}$ & -0.041 & -0.041 & -0.039 & -0.045 \\
$P_{33}$ & 0.178 & 0.187 & 0.209 & 0.214 \\
\hline \hline
\end{tabular}

TABLE III. Scattering lengths and volumes obtained from the BS equation in units of $m_{\pi}^{-(2 \ell+1)}$, compared to results from the SM95 [33] and KH80 [58] $\pi N$ partial wave analyses.

\begin{tabular}{llllll}
\hline \hline$f_{\pi N \Delta}^{2} / 4 \pi$ & $x_{\Delta}$ & $g_{\rho}^{2} / 4 \pi$ & $\kappa_{\rho}$ & equation & ref. \\
\hline 0.365 & -0.11 & 2.88 & 2.66 & BS (RS) & this work \\
0.741 & - & 2.73 & 4.26 & BS (Pas) & this work \\
0.35 & -0.3 & 2.5 & 3.7 & $\mathrm{ET}$ & {$[17]$} \\
0.43 & - & 2.85 & 1.8 & $\mathrm{ET}$ & $[1]]$ \\
0.36 & -0.12 & 3.13 & 2.25 & $\mathrm{Sm}$ & {$[[1]]$} \\
0.36 & -0.41 & 2.90 & 1.44 & $\mathrm{BbS}$ & {$[[1]]$} \\
0.40 & -0.21 & 3.36 & 6.6 & $\mathrm{Ka}$ & {$[11]$} \\
0.36 & -0.31 & 3.03 & 3.16 & $\mathrm{Tr}$ & {$[11]$} \\
0.36 & - & 3.1 & 2.7 & $\mathrm{Tr}$ & {$[18]$} \\
\hline \hline
\end{tabular}

TABLE IV. Comparison between coupling constants obtained from the Bethe-Salpeter (BS), equal-time (ET), smooth (Sm), Blankenbecler-Sugar (BbS), Kadyshevsky (Ka) equations, and tree-level (Tr) calculations. 


\begin{tabular}{lrrrr}
\hline \hline coupling constants & $n_{\pi}=2$ & $n_{\pi}=4$ & $n_{\pi}=10$ & model I (RS) \\
\hline$g_{\pi N N}^{2} / 4 \pi$ & 13.5 & 13.5 & 13.5 & 13.5 \\
$g_{\pi N N}^{(0) 2} / 4 \pi$ & 4.23 & 4.68 & 5.98 & 1.80 \\
$f_{\pi N \Delta}^{2} / 4 \pi$ & $\mathbf{0 . 3 6 5}$ & $\mathbf{0 . 3 6 5}$ & $\mathbf{0 . 3 7 1}$ & $\mathbf{0 . 3 6 5}$ \\
$f_{\pi N \Delta}^{(0) 2} / 4 \pi$ & $\mathbf{0 . 1 7}$ & $\mathbf{0 . 2 0}$ & $\mathbf{0 . 1 9 6}$ & $\mathbf{0 . 3 7}$ \\
$x_{\Delta}$ & $\mathbf{- 0 . 1 3}$ & $\mathbf{- 0 . 2 4}$ & $\mathbf{- 0 . 1 8}$ & $\mathbf{0 . 1 1}$ \\
$g_{\rho}^{2} / 4 \pi$ & $\mathbf{2 . 6 7}$ & $\mathbf{2 . 6 3}$ & $\mathbf{2 . 8 0}$ & $\mathbf{2 . 8 8}$ \\
$\kappa_{\rho}$ & $\mathbf{2 . 1 8}$ & $\mathbf{2 . 0 3}$ & $\mathbf{2 . 1 5}$ & $\mathbf{2 . 6 6}$ \\
$g_{\sigma \pi \pi} g_{\sigma N N} / 4 \pi$ & $\mathbf{0 . 8 6}$ & $\mathbf{0 . 3 9}$ & $\mathbf{0 . 4 8}$ & $\mathbf{0 . 4 1}$ \\
\hline$m_{a s s e s}^{(0)}$ & & & & 1.34 \\
$m_{N}^{(0)}$ & 1.18 & 1.14 & 1.11 & $\mathbf{2 . 3 0 5}$ \\
$m_{\Delta}$ & $\mathbf{1 . 4 9 5}$ & $\mathbf{1 . 4 9 2}$ & $\mathbf{1 . 4 3 5}$ & $\mathbf{0 . 6 5}$ \\
$m_{\sigma}$ & $\mathbf{0 . 8 8}$ & $\mathbf{0 . 6 2}$ & $\mathbf{0 . 6 4}$ & $\mathbf{1 . 7 7}$ \\
$\Lambda_{\pi}$ & $\mathbf{1 . 3 4}$ & $\mathbf{1 . 8 5}$ & $\mathbf{2 . 7 3}$ & \\
\hline \hline
\end{tabular}

TABLE V. The coupling constants and particle masses resulting from fits to the $\pi N$ data using different values of $n_{\pi}$. The parameters from the model I fit using the Rarita-Schwinger propagator are also shown for comparison. The quantities in boldface were varied in the fits. All masses are in GeV. Particle masses not given are the same as those given in Table [1].

\begin{tabular}{rrrr}
\hline \hline & $n_{\pi}=2$ & $n_{\pi}=4$ & $n_{\pi}=10$ \\
\hline$\Lambda_{\pi}^{R}$ & 0.874 & 0.868 & 0.822 \\
$\Delta_{\pi}$ & $2.47 \%$ & $2.51 \%$ & $2.79 \%$ \\
\hline \hline
\end{tabular}

TABLE VI. The renormalized pion cutoff masses $\Lambda_{\pi}^{R}$ (in GeV) and values of $\Delta_{\pi}$ (expressed as percentages). 


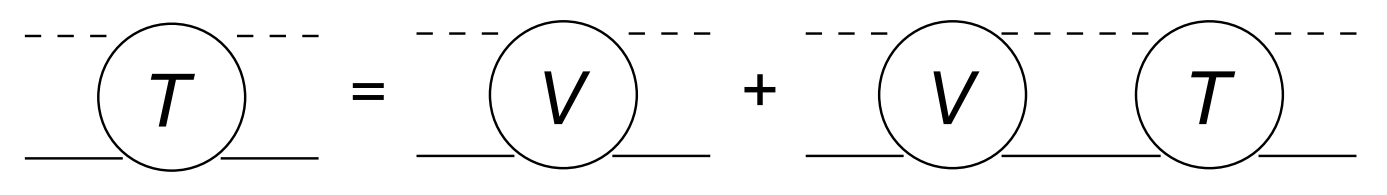

$$
\left.V=V_{\mathbb{N P}}+\sum_{B} \Gamma_{\Gamma_{B}^{(0)}} \Gamma_{\sigma^{(0)}}\right)^{-\cdots}
$$

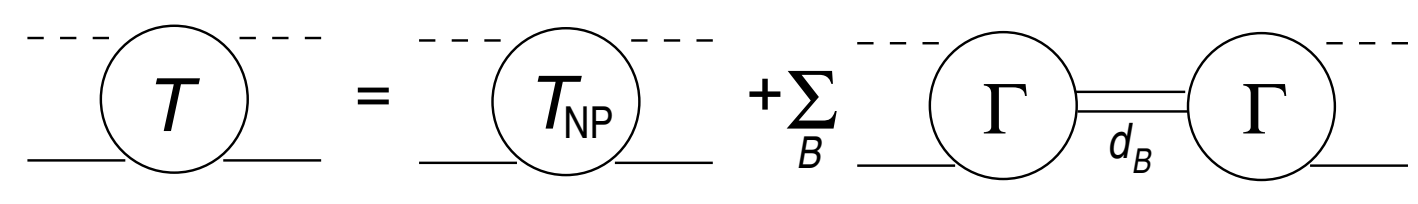

$$
\left.\left(T_{\mathrm{NP}}\right)^{--}=\mathrm{V}_{\mathrm{NP}}+\mathrm{V}_{\mathrm{NP}}\right)^{---}\left(T_{\mathrm{NP}}\right)^{--}
$$

FIG. 1. Graphical representation of: (a) the BS equation for the full $T$-matrix, (b) the potential and (c) the $T$-matrix in terms of non-pole and pole parts, and (d) the BS equation for the non-pole $T$-matrix.

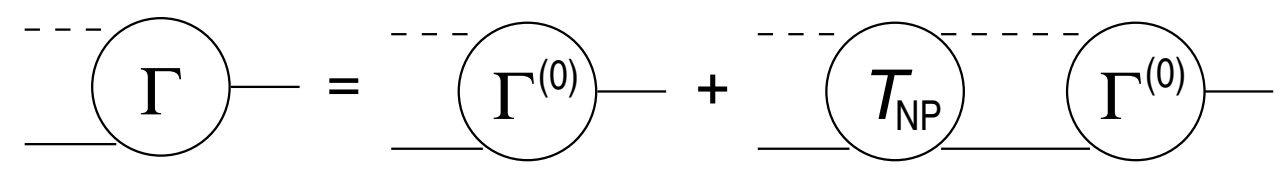

FIG. 2. The equation for the dressed $\pi N B$ vertex.

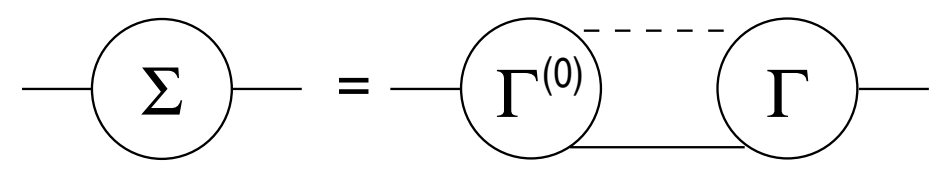

FIG. 3. The baryon self-energy. 

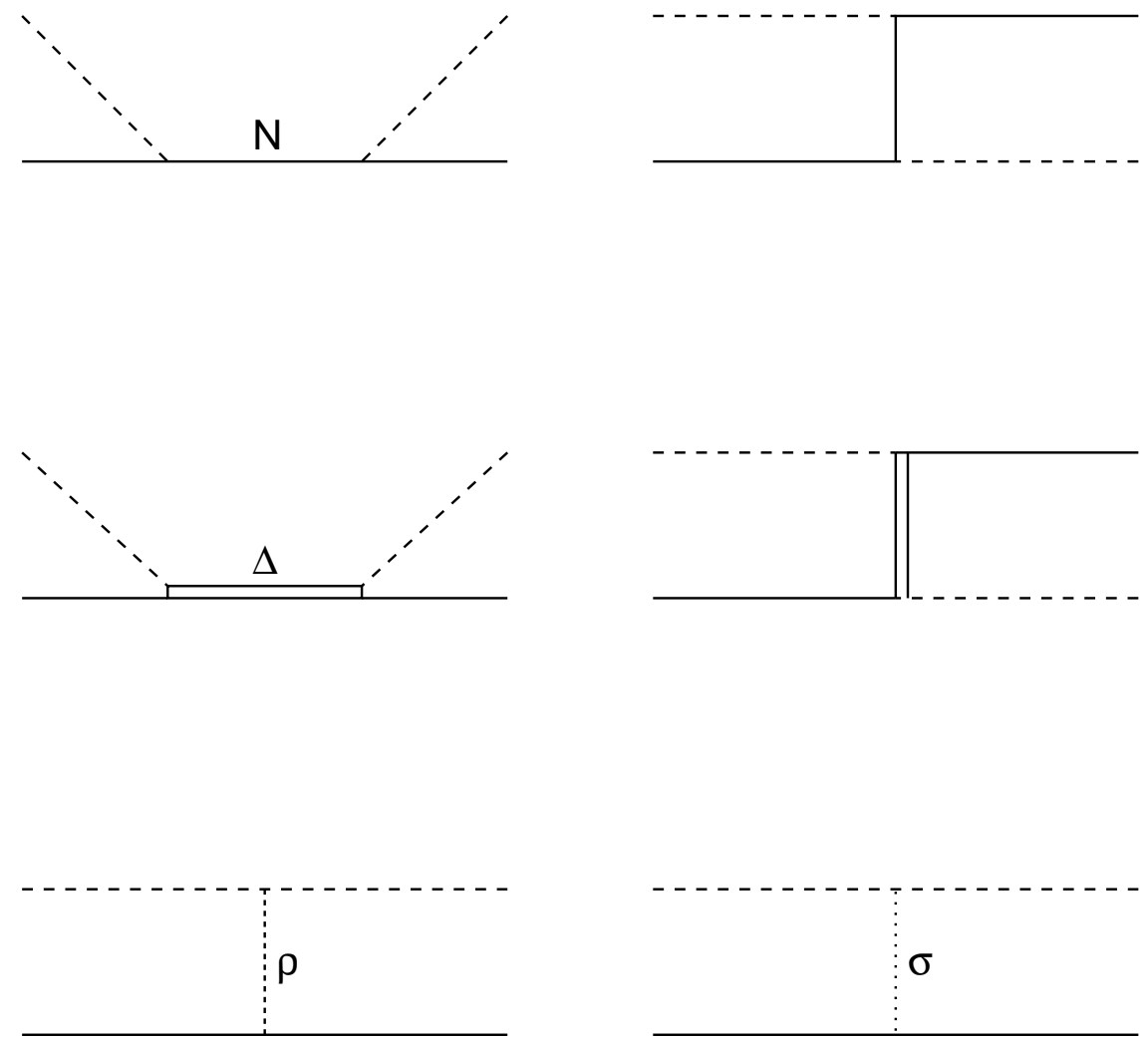

FIG. 4. The diagrams included in the potential of the BS equation. 

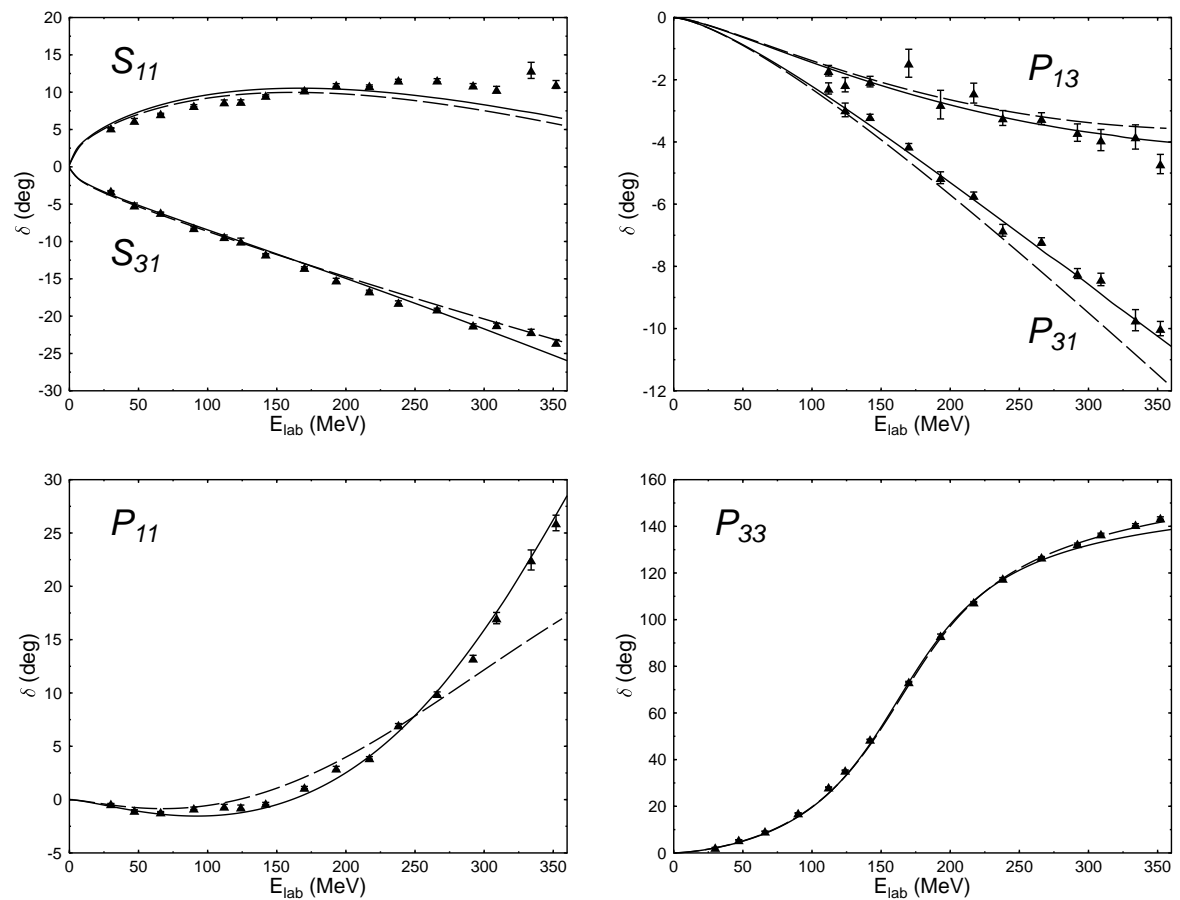

FIG. 5. The phase shifts obtained from the BS equation shown versus the pion laboratory energy, using the Rarita-Schwinger $\Delta$ propagator (-) and Pascalutsa $\Delta$ propagator (--). Data points from the VPI SM95 partial wave analysis are also shown. 

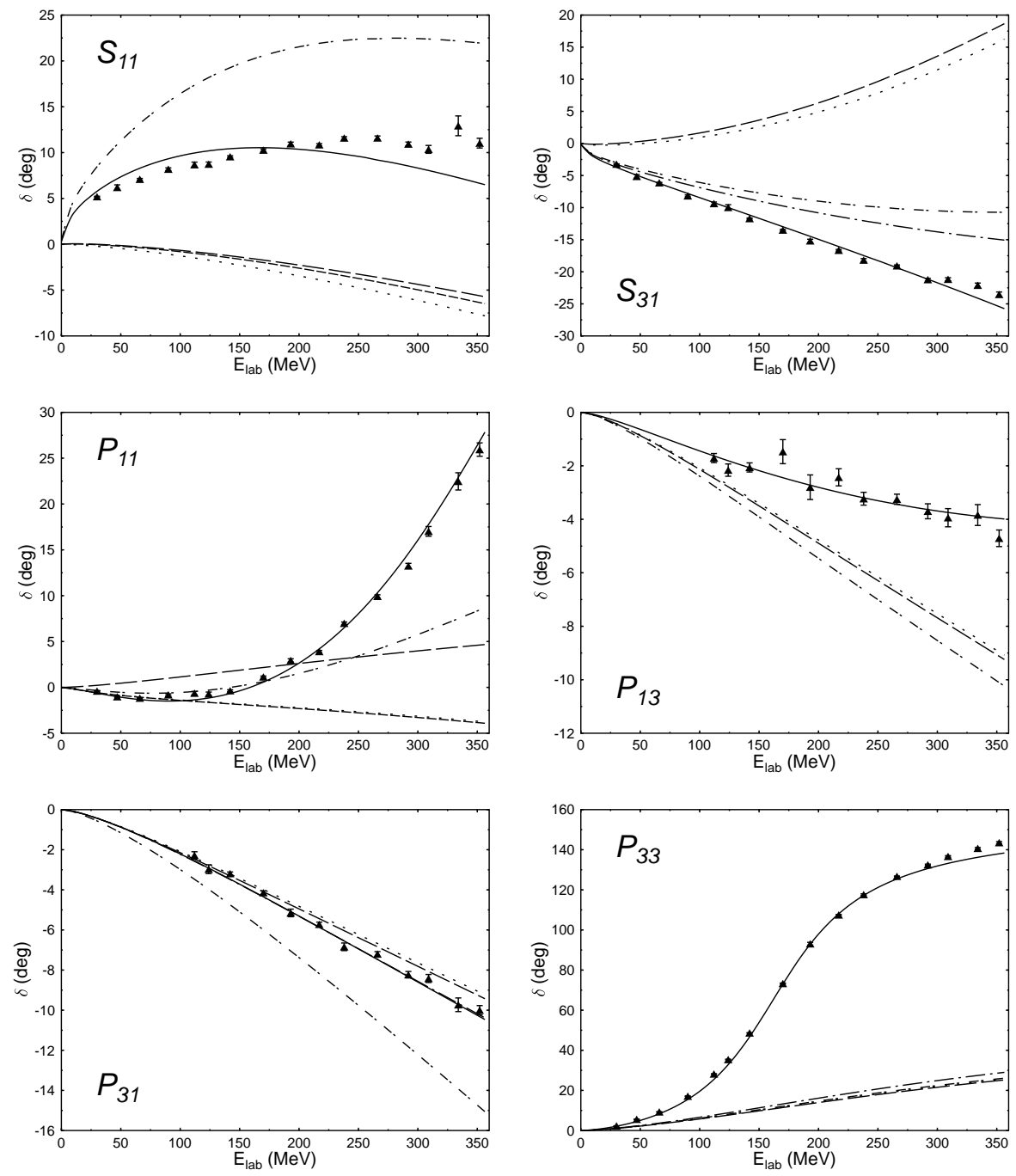

FIG. 6. Contributions to the $\pi N$ phase shifts as each diagram is added to the potential, in the following order: $u$-channel $N$ pole $(--), s$-channel $N$ pole $(--), t$-channel $\sigma$ exchange $(\cdots), t$-channel $\rho$ exchange $(-\cdot), u$-channel $\Delta$ pole $(--)$, and $s$-channel $\Delta$ pole $(-)$. 

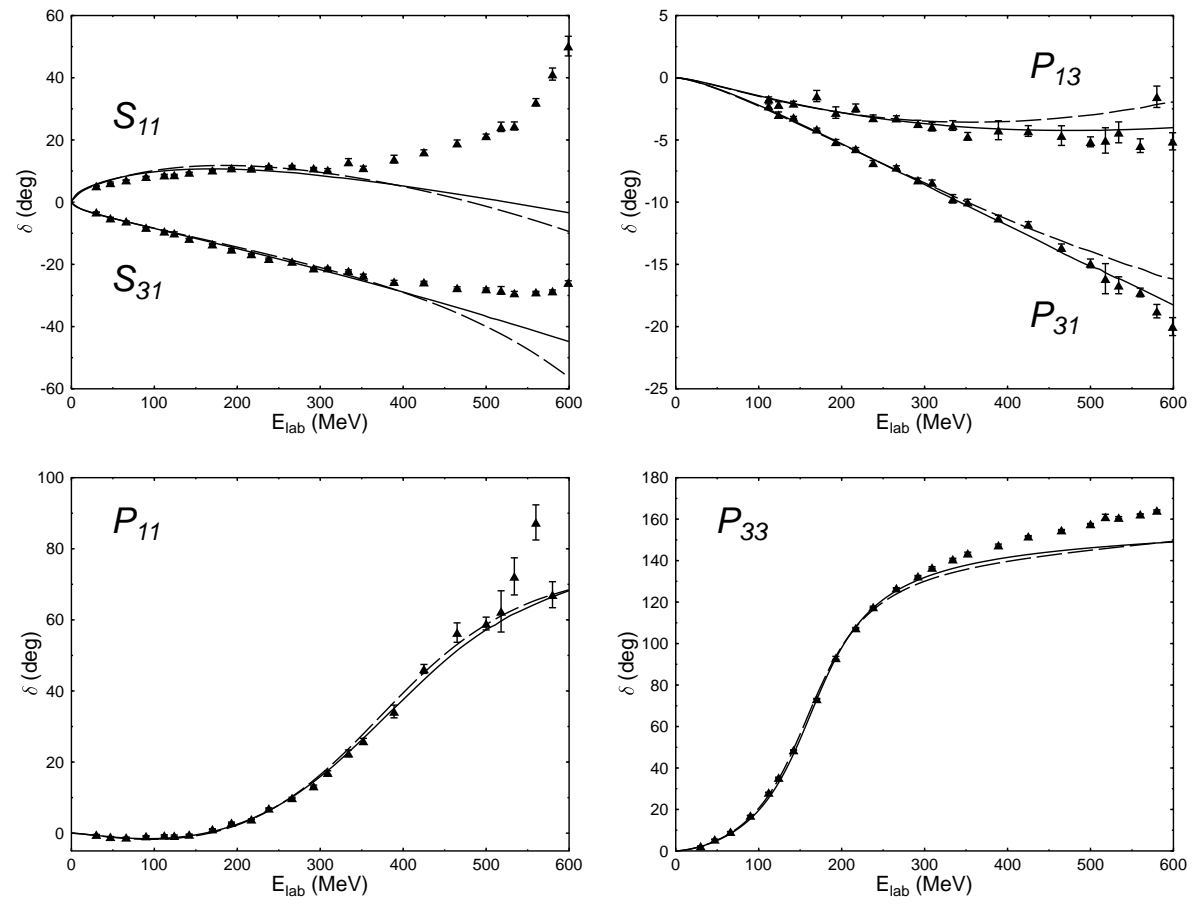

FIG. 7. The phase shifts obtained from the BS equation are shown versus the pion laboratory energy up to $600 \mathrm{MeV}$, for models I (-) and II (--). 